EPJ manuscript No.

(will be inserted by the editor)

\title{
Inclusive $\mathrm{K}^{+}$-meson production in proton-nucleus interactions
}

\author{
M. Büscher ${ }^{1 a}$, V. Koptev ${ }^{2}$, M. Nekipelov ${ }^{1,2}$, Z. Rudy ${ }^{3}$, H. Ströher ${ }^{1}$, Yu. Valdau ${ }^{1,2}$, S. Barsov², M. Hartmann ${ }^{1}$, \\ V. Hejny ${ }^{1}$, V. Kleber ${ }^{4}$, N. Lang ${ }^{5}$, I. Lehmann ${ }^{1,6}$ b, S. Mikirtychiants ${ }^{2}$, and H. Ohm ${ }^{1}$ \\ 1 Institut für Kernphysik, Forschungszentrum Jülich, 52425 Jülich, Germany \\ 2 High Energy Physics Department, Petersburg Nuclear Physics Institute, 188350 Gatchina, Russia \\ 3 Institute of Physics, Jagellonian University, Reymonta 4,30059 Cracow, Poland \\ 4 Institut für Kernphysik, Universität zu Köln, Zülpicher Str. 77, 50937 Köln, Germany \\ 5 Institut für Kernphysik, Universität Münster, W.-Klemm-Str. 9, 48149 Münster, Germany \\ ${ }^{6}$ Institut für Hadronen- und Kernphysik, Forschungszentrum Rossendorf, D-01474 Dresden \\ Received: date / Revised version: date
}

\begin{abstract}
The production of $K^{+}$-mesons in $p A(A=\mathrm{D}, \mathrm{C}, \mathrm{Cu}, \mathrm{Ag}, \mathrm{Au})$ collisions has been investigated at the COoler SYnchrotron COSY-Jülich for beam energies $T_{p}=1.0-2.3 \mathrm{GeV}$. Double differential inclusive $p \mathrm{C}$ cross sections at forward angles $\vartheta_{K^{+}}<12^{\circ}$ as well as the target-mass dependence of the $K^{+}$momentum spectra have been measured with the ANKE spectrometer. Far below the free $N N$ threshold at $T_{N N}=1.58 \mathrm{GeV}$ the spectra reveal a high degree of collectivity in the target nucleus. From the targetmass dependence of the cross sections at higher energies, the repulsive in-medium potential of $K^{+}$-mesons can be deduced. Using $p N$ cross-section parameterisations from literature and our measured $p \mathrm{D}$ data we derive a cross-section ratio of $\sigma\left(p n \rightarrow K^{+} X\right) / \sigma\left(p p \rightarrow K^{+} X\right) \sim(3-4)$.
\end{abstract}

PACS. 13.75.-n Hadron-induced low- and intermediate-energy reactions and scattering - 25.40.-h Nucleoninduced reactions and scattering

\section{Overview}

One important topic of contemporary hadron physics is the investigation of the influence of the nuclear medium on the properties of hadrons and their production processes. Such phenomena can, for example, be studied in detail by measuring meson production in proton-nucleus collisions. If the measurements are carried out at projectile energies below the threshold for free nucleon-nucleon $(N N)$ collisions (so-called subthreshold production) these processes necessarily imply collective effects involving several of the nucleons inside the target nucleus. $K^{+}$-production is particularly well suited for such investigations since this meson is heavy compared to the pion, so that its production requires strong medium effects. As a consequence, the production of $\mathrm{K}^{+}$-mesons in proton-nucleus collisions is an appropriate tool to learn either about cooperative nuclear phenomena or high momentum components in the nuclear many-body wave function.

In this paper we present data obtained at the ANKE spectrometer [1] of COSY-Jülich on inclusive $K^{+}$production with various targets and over a wide range of beam energies, see Sects. 3 and 5. A simple parameterisation, in terms of a few Lorentz-invariant variables, is presented

\footnotetext{
am.buescher@fz-juelich.de

b Present address: Department of Radiation Sciences, University of Uppsala, Box 535, S-75121 Uppsala, Sweden
}

in Sect. 4 which allows us to compare our data, measured at near-forward angles $\vartheta<12^{\circ}$, with those obtained in other experiments under different kinematical conditions. It is shown that the data at the lowest beam en$\operatorname{ergy} T_{p}=1.0 \mathrm{GeV}[2]$, reveal a large collective behaviour of the target nucleons. Either large intrinsic momenta of the nucleons must be involved in the $K^{+}$-formation processes or the number of participating nucleons must be significantly larger than one. The latter could, for example, be due to multi-step processes. At the higher energies, $T_{p} \geq 2.0 \mathrm{GeV}$, our data suggest that direct kaon production on single nucleons prevails.

Final state interactions (FSI) can mask the information about the production mechanisms. However, due to the strangeness content of the $K^{+}$-meson $(S=+1)$, FSI effects are generally considered to be rather small. As a consequence the mean free path of $K^{+}$-mesons at normal nuclear density $\rho_{0}$ is as large as $\sim 6 \mathrm{fm}$. In a previous publication [3] we have shown that the low momentum part of the $K^{+}$-spectrum is sensitive to the Coulomb and the in-medium repulsive nuclear potential. In Sect. 3.2 we compare the data from ANKE with model calculations using a CBUU transport code $[4,5]$. The comparison allows for a determination of the $\mathrm{K}^{+}$-potential at normal nuclear density.

Experimental data on the $K^{+}$-production cross section from proton-neutron $(p n)$ interactions in the close- 
to-threshold regime are not yet available. This quantity is crucial, for example, for the theoretical description of proton-nucleus $(p A)$ and nucleus-nucleus $(A A)$ data. Predictions for the ratio of the proton-induced production cross sections on neutrons $\left(\sigma_{n}\right)$ and protons $\left(\sigma_{p}\right), R_{n p}=$ $\sigma_{n} / \sigma_{p}$, range from unity to a factor of six, depending on the underlying model assumptions. In Ref. [6] it was proposed that there should be no difference between $K^{+}$production on the neutron and proton, whereas isospin-based models yield $R_{n p} \sim 2$ [7]. The authors of Ref. [8] draw an analogy between $K^{+}$- and $\eta$-meson production and predict $R_{n p} \sim 6$. In Sect. 5 we present inclusive ANKE data for $K^{+}$production in $p$ D reactions. Based on a simple phasespace estimate we conclude that the total $K^{+}$-production cross section on neutrons is significantly larger than that on protons.

\section{$2 K^{+}$identification with ANKE and determination of absolute cross sections}

\subsection{The ANKE spectrometer and $K^{+}$detectors}

The COoler SYnchrotron COSY-Jülich [9], which provides proton beams in the range $T_{p}=0.04-2.88 \mathrm{GeV}$, is well suited for the study of $K^{+}$-meson production in $p p$ and $p A$ reactions. In measurements with very thin windowless internal targets, secondary processes of the produced mesons can be neglected and, simultaneously, sufficiently high luminosities are obtained. Foil or cluster-jet targets have been used at ANKE, providing effective luminosities of $\mathcal{L} \gtrsim 10^{32} \mathrm{~cm}^{-2} \mathrm{~s}^{-1}$ and $\mathcal{L} \gtrsim 10^{31} \mathrm{~cm}^{-2} \mathrm{~s}^{-1}$, respectively. For the foil-target measurements, the COSY beam with an intensity of $(2-4) \times 10^{10}$ protons is accelerated to the desired energy on an orbit below the target (in order to avoid unwanted beam-target interactions and beam losses during acceleration) [1]. These targets are strips of $\mathrm{C}, \mathrm{Cu}, \mathrm{Ag}$ or $\mathrm{Au}$ with thicknesses of $(40-1500) \mu \mathrm{g} / \mathrm{cm}^{2}$. After acceleration the beam is slowly steered upward onto the target over a period of approximately $50 \mathrm{~s}$, keeping the trigger rate in the detectors nearly constant at (1000$1500) \mathrm{s}^{-1}$, a level that can be handled by the data acquisition system with dead time corrections of less than $25 \%$. For the study of $p \mathrm{D}$ interactions, a deuterium cluster-jet target [10] has been used, providing areal densities of up to $\sim 5 \times 10^{14} \mathrm{~cm}^{-2}$. Due to the lower target density, the cycle times were here typically in the range of 5-30 minutes, and the rate load on the detectors was smaller than in the case of the solid targets.

When using the deuterium cluster-jet target at ANKE, the luminosity can be measured with a telescope consisting of three silicon counters with thicknesses of respectively $60 \mu \mathrm{m}, 300 \mu \mathrm{m}$, and $5 \mathrm{~mm}$ [11]. These so called "spectator" counters are located within the vacuum pipe of the accelerator a few centimetres to the side of the circulating proton beam, see Fig. 1. They cover polar angles in the range $83^{\circ}-104^{\circ}$ and $\pm 7^{\circ}$ in azimuth. The ability to identify a deuteron and measure its kinetic energy in the telescope allows one to determine the luminosity by measuring proton-deuteron elastic scattering in parallel to kaon production. Such a "direct" luminosity measurement is not possible with the foil targets since the differential cross sections for the emission of spallation products are not known with sufficient accuracy. Therefore, a different procedure has been developed where the measured $K^{+}$yields from the carbon target are normalised to pion cross sections from literature, see Sect. 2.2. A major advantage of this method is that only the ratios of luminosities $\mathcal{L}_{\pi^{+}} / \mathcal{L}_{K^{+}}$during $\pi^{+}$calibration and $K^{+}$data-taking runs has to be determined, which reduces systematic uncertainties.

Subthreshold $\mathrm{K}^{+}$-production was one of the primary motivations for building the large-acceptance ANKE spectrometer [1]. This facility consists of three dipole magnets, which separate forward-emitted reaction products from the circulating proton beam and allow one to determine their emission angles and momenta. Depending on the choice of the magnetic field in the spectrometer dipole D2 (see Fig. 1), $K^{+}$-mesons in the momentum range $p_{K} \sim 150-600 \mathrm{MeV} / \mathrm{c}$ can be detected. The angular acceptance for these momenta is $\pm 12^{\circ}$ horizontally and up to $\pm 7^{\circ}$ vertically.

The layout of ANKE, including detectors and the dataacquisition system, has been optimised to study $K^{+}$spectra at beam energies down to $T_{p} \sim 1.0 \mathrm{GeV}[2,12]$. This is a very challenging task because of the smallness of the $K^{+}$production cross sections, e.g. $39 \mathrm{nb}$ for $p \mathrm{C}$ collisions at $1.0 \mathrm{GeV}$ [13]. The ANKE detection system for $K^{+}$mesons, located along the side of D2, permits kaon identification in a background of pions, protons and scattered particles up to $10^{6}$ times more intense. For the identification of the $K^{+}$-mesons the following criteria are used:

- ANKE provides horizontal momentum focusing of the ejectiles. This is used to define the $K^{+}$-momenta in 15 telescopes which are located along the focal surface of the spectrometer dipole D2. The momentum ranges covered by each telescope, given in the left columns of Tables 4 and 8 in the Appendix, are determined by their widths $(10 \mathrm{~cm})$ and the dispersion of D2.

- A fast on-line trigger system, based on start- and stop detectors of a time-of-flight setup, allows for the selection of individual start-stop combinations and thus of particles with certain momenta and emission angles at the target. Narrow time-of-flight (TOF) gates can be defined for the identification of pions, kaons and protons, respectively. 23 TOF-start counters are located close to the exit window of the D2 vacuum chamber in order to maximise the distance to 15 TOF-stop counters which are the foremost ones in the telescopes (see Fig. 1).

- The range telescopes discriminate pions, kaons and protons with the same momenta due to their different energy losses. Passive copper degraders in the telescopes between the scintillation counters enhance the discrimination efficiency.

- The $K^{+}$-mesons are stopped in the $\Delta E$ counters or in the second degrader of each telescope. Their decay mainly into $\mu^{+} \nu_{\mu}$ and $\pi^{+} \pi^{0}$ with a lifetime of 

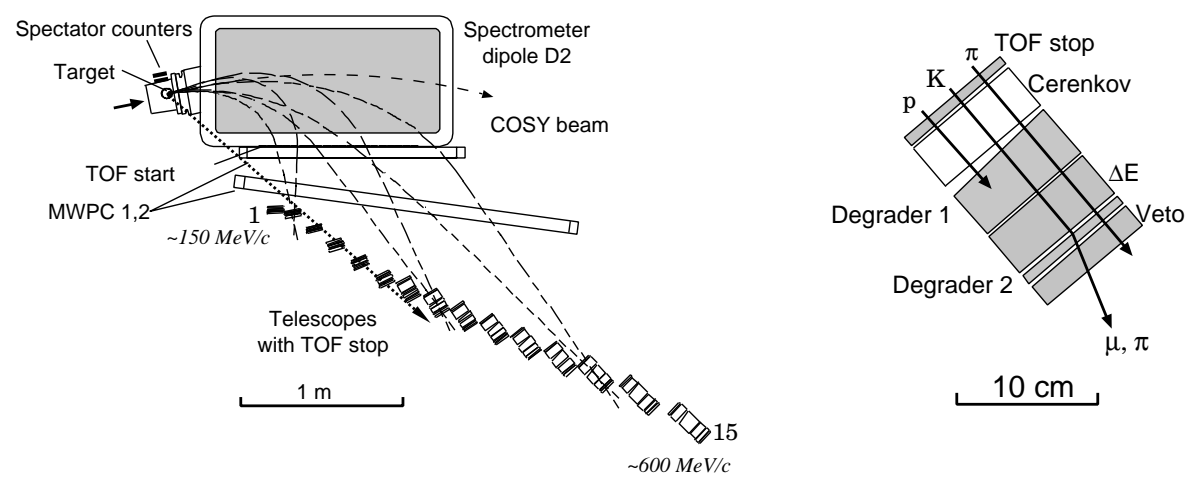

Fig. 1. Layout of ANKE and the $K^{+}$-detection system. The two MWPCs provide tracking and momentum reconstruction of the ejectiles. $K^{+}$-identification is achieved with the help of time-of-flight (TOF) and energy-loss measurements in the 15 range telescopes [12]. The momentum range covered by the telescopes is indicated for the maximum field strength in D2 of $B=1.57 \mathrm{~T}$. The dashed lines depict trajectories of particles with low/medium/high momenta and horizontal emission angles of $\pm 10^{\circ}$ at the target. The dotted line sketches the trajectory of particles bypassing D2 and hitting stop counters 2-5, which are used for luminosity monitoring. Also indicated is the location of the target-near spectator counters for luminosity monitoring together with the cluster target. On the right hand side a schematic top view of one telescope is presented.

$\tau=12.4$ ns provides a very effective criterion for kaon identification via the detection of delayed signals in a so-called veto counter (with respect to prompt signals from e.g. a $\pi^{+}$produced in the target punching through all counters of a telescope).

- The tracks of the ejectiles are measured with two multiwire proportional chambers (MWPCs). From this information the emission angles at the target are deduced and a suppression of background not originating from the target is possible.

For further details of the $K^{+}$identification procedures, we refer to an earlier publication [12].

The production of $\mathrm{K}^{+}$-mesons has been studied with ANKE at beam energies $T_{p}=1.0,1.2,1.5,2.0$ and $2.3 \mathrm{GeV}$ for $\mathrm{C}, \mathrm{Cu}, \mathrm{Ag}$ and $\mathrm{Au}$ targets. Two different settings of the magnetic field in D2 were used: $B=1.3 \mathrm{~T}$ ("1.3 T mode") for $T_{p}=1.0,1.2,2.0$ and $2.3 \mathrm{GeV}$, and $B=$ $1.57 \mathrm{~T}$ ("1.6 T mode") for $T_{p}=1.2,1.5$ and $2.3 \mathrm{GeV}$. With these two modes of operation different regions of the kaon-momentum spectra can be explored. For example, the lower D2 magnetic field of $B=1.3 \mathrm{~T}$ is sufficient to measure the complete kaon momentum spectrum at $T_{p}=1.0 \mathrm{GeV}$, whereas with the $1.6 \mathrm{~T}$ mode larger kaon momenta are accessible, which is advantageous at higher beam energies. The geometry of the experimental setup as well as the $K^{+}$-identification procedures is identical for the two field values, though different methods for the normalisation to pion cross sections have been applied. The $K^{+}$spectra from $p \mathrm{D}$ interactions have been obtained at $T_{p}=2.02 \mathrm{GeV}$ using the $1.6 \mathrm{~T}$ mode, and for $T_{p}=1.83$ $\mathrm{GeV}$ at an intermediate field strength ("1.45 T mode").

\subsection{Normalisation of the $p \mathrm{C} \rightarrow K^{+} X$ cross sections}

When using foil targets at ANKE, absolutely normalised kaon cross sections are deduced from the number of identified kaons $N_{K^{+}}^{i}$ in each telescope $i$ (with mean momenta $p^{i}$ ), using differential $\pi^{+}$cross sections from literature measured under similar kinematical conditions.

The detection and identification of $\pi^{+}$-mesons, which have been measured in different runs with dedicated trigger conditions, in the ANKE detectors is rather simple and the analysis procedures are straightforward. Since the pions can be identified by TOF and energy-loss in the stop counters alone, the pion detection efficiency is given by

$$
\epsilon_{\pi^{+}}^{i}=\epsilon_{\pi^{+}}^{\mathrm{TOF}(i)} \cdot \epsilon_{\pi^{+}}^{\mathrm{MWPC}(i)} \cdot \epsilon_{\pi^{+}}^{\text {decay }}
$$

The efficiency of the TOF- $\Delta E$ criterion for pions is large, $\epsilon_{\pi^{+}}^{\mathrm{TOF}(i)} \geq 98 \%$ [12]. The MWPC efficiencies $\epsilon_{\pi^{+}}^{\mathrm{MWPC}(i)}$ have been measured for each beam energy and are larger than 95\%. The decay probabilities between target and stop counters were obtained from simulation calculations.

The differential cross sections for $\pi^{+}$-production in $p \mathrm{C}$ interactions have been taken from Refs. $[14,15]$. The $\pi^{+}$ momentum spectrum has been measured at $T_{p}=1.0 \mathrm{GeV}$ for $\vartheta_{\pi^{+}}=0^{\circ}$ at the Petersburg Nuclear Physics Institute (PNPI) [14]. At higher beam energies only the production cross sections at a fixed pion momentum of $p_{\pi}=500$ $\mathrm{MeV} / \mathrm{c}$ and $\vartheta_{\pi}=2.5^{\circ}$ are known from an experiment at the Lawrence Berkeley Laboratory (LBL) for $T_{p}=$ $1.05,1.73,2.1$ and $4.2 \mathrm{GeV}$, both for positively and negatively charged pions [15]. The results of the two experiments have to be combined before they can be used for the normalisation of the ANKE data. Additional values for the $\pi^{+}$-production cross section at $T_{p}=2.66$ and $3.5 \mathrm{GeV}$ can be calculated when cross sections of $\pi^{-}$-production, which have been measured at LBL also for these intermediate energies [15], are included into the analysis.

The ratios of the differential pion cross sections $R=$ $\sigma_{\pi^{+}} / \sigma_{\pi^{-}}$calculated from the LBL data [15] for $T_{p}=$ 1.05, 1.73, 2.1 and $4.2 \mathrm{GeV}$ are shown in Fig. 2 (upper). When fitting these ratios by a polynomial, only the statistical errors of the data have been taken into account in order to avoid double counting the systematic errors, 
which are considered later. The resulting interpolated values of $R$ at $T_{p}=2.66$ and $3.5 \mathrm{GeV}$ together with the $\pi^{-}$production cross sections from Ref. [15], can then be used to derive the $\pi^{+}$cross sections at these energies. This gives $\mathrm{d}^{2} \sigma_{\pi^{+}} / \mathrm{d} \Omega \mathrm{d} p=(41.8 \pm 1.2)$ and $(59.8 \pm 1.2) \mu \mathrm{b} /(\mathrm{sr} \mathrm{MeV} / \mathrm{c})$ at $T_{p}=2.66$ and $3.5 \mathrm{GeV}$, respectively.
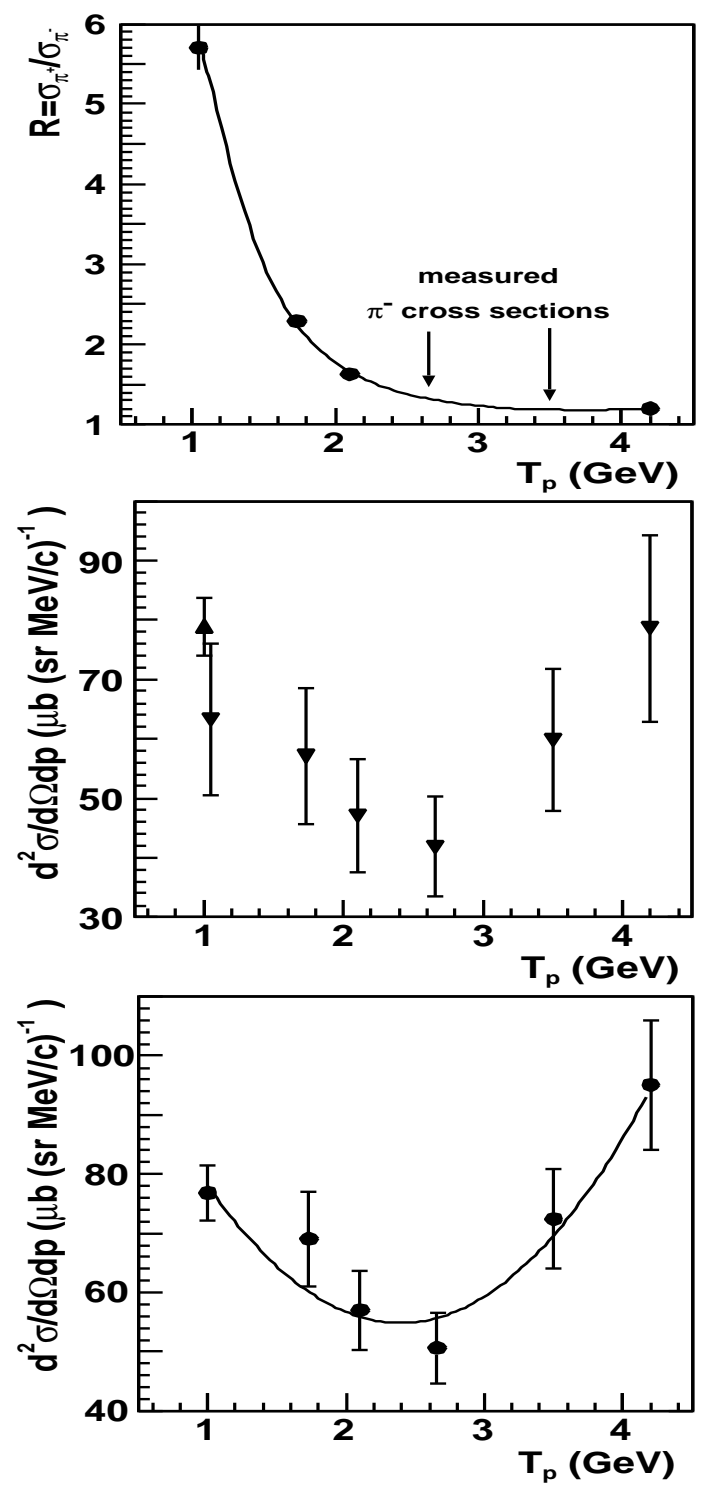

Fig. 2. Upper: Ratio of pion-production cross sections $R=$ $\sigma_{\pi^{+}} / \sigma_{\pi^{-}}$for $p C \rightarrow \pi^{ \pm} X$ reactions at $p_{\pi}=500 \mathrm{MeV} / \mathrm{c}$ as a function of the beam energy. The solid dots represent the calculated ratios using $\pi^{+}$and $\pi^{-}$cross sections from Ref. [15]. The solid line is a polynomial fit to these points. The energies where only $\pi^{-}$cross sections are available [15] are indicated. Middle: $\pi^{+}$-production cross sections at $p_{\pi}=500 \mathrm{MeV} / \mathrm{c}$. Upwards pointing triangles denote the data from Ref. [14], downwards represent the data from Ref. [15]. Lower: Averaged $\pi^{+}$ production cross sections as a function of beam energy. The curve shows the result of the fit with second order polynomial. See text for definition of the error bars.
The combined cross sections for $\pi^{+}$production are shown in Fig. 2 (middle). In Ref. [14] a total systematic uncertainty of $6 \%$ is given, while the authors of Ref. [15] indicate an overall systematic uncertainty of $20 \%$ and a relative systematic uncertainty between the individual data points of $\sim 10 \%$. The mean value of the PNPI and LBL cross sections at $T_{p}=1.0 \mathrm{GeV}$ of $(76.9 \pm 4.6) \mu \mathrm{b} /(\mathrm{sr} \mathrm{MeV} / \mathrm{c})$ shown in Fig. 2 (lower) has been calculated according to the averaging method from PDG [16] for results of different experiments (i.e. for averaging and fitting the statistical and systematic errors are added in quadrature and this combined error is used later on). The cross sections from Ref. [15] for $T_{p}=1.05,1.73,2.1,2.66,3.5$ and $4.2 \mathrm{GeV}$ have been scaled up in order to match the averaged value at $T_{p}=1.0 \mathrm{GeV}$ (Fig. 2 (lower)). The total error of these points is the quadratic sum of the above mentioned relative systematic uncertainty and the error of the scaling factor.

In order to determine the $\pi^{+}$cross section at any intermediate energy, the data have been fitted by a secondorder polynomial. The error of the fit has to take into account a complete error matrix since the systematic uncertainties of the data are the main source of the errors. The result of this fitting procedure is presented in Fig. 2 (lower) and Table 1.

Table 1. $\pi^{+}$-production cross sections for $p_{\pi^{+}}=500 \mathrm{MeV} / \mathrm{c}$ as a function of the beam energy calculated from the data in Refs. [14,15]. The errors include all statistical and systematic errors, as explained in the text.

\begin{tabular}{cc}
\hline$T_{p}(\mathrm{GeV})$ & $\frac{\mathrm{d}^{2} \sigma}{\mathrm{d} \Omega \mathrm{d} p}\left(\frac{\mu \mathrm{b}}{\mathrm{srMeV} / \mathrm{c}}\right)$ \\
\hline 1.0 & $78.1 \pm 4.6$ \\
1.2 & $71.9 \pm 3.9$ \\
1.5 & $64.4 \pm 3.5$ \\
2.0 & $56.7 \pm 4.0$ \\
2.3 & $55.0 \pm 4.2$ \\
\hline
\end{tabular}

In the following the notation $\sigma \equiv \mathrm{d}^{2} \sigma /(\mathrm{d} \Omega \mathrm{d} p)$ is used for the differential kaon (pion) cross sections which can be calculated from $N_{K^{+}}^{i}\left(N_{\pi^{+}}^{i}\right)$ as

$$
\begin{gathered}
\sigma_{K^{+}}\left(T_{p}, p^{i}\right)=\frac{N_{K^{+}}^{i}\left(T_{p}\right)}{(\Delta p \Delta \Omega)_{K^{+}}^{i}} \cdot \frac{1}{\mathcal{L}_{K^{+}}\left(T_{p}\right)} \cdot \frac{1}{\epsilon_{K^{+}}^{i}} \\
\sigma_{\pi^{+}}\left(T_{p}, p^{i}\right)=\frac{N_{\pi^{+}}^{i}\left(T_{p}\right)}{(\Delta p \Delta \Omega)_{\pi^{+}}^{i}} \cdot \frac{1}{\mathcal{L}_{\pi^{+}}\left(T_{p}\right)} \cdot \frac{1}{\epsilon_{\pi^{+}}^{i}},
\end{gathered}
$$

where $(\Delta p \Delta \Omega)^{i}$ are the angular-momentum bins covered by each telescope and $\mathcal{L}_{K^{+}}\left(\mathcal{L}_{\pi^{+}}\right)$denote the luminosities integrated over the corresponding data-taking runs. Similar to pions the kaon-identification efficiencies are given by

$$
\epsilon_{K^{+}}^{i}=\epsilon_{K^{+}}^{\text {Tel. }(i)} \cdot \epsilon_{K^{+}}^{\mathrm{MWPC}(i)} \cdot \epsilon_{K^{+}}^{\text {decay }} .
$$

The efficiencies of the individual telescopes are in the range $\epsilon_{K^{+}}^{\text {Tel. }(i)} \sim 10-30 \%$. They have been obtained from 
a dedicated calibration run at $T_{p}=2.3 \mathrm{GeV}[12]$. The MWPC efficiencies amount to $\epsilon_{K^{+}}^{\mathrm{MWPC}(i)}=71-93 \%[17]$ depending on the kaon momenta, i.e. the telescope number $i$.

From the analysis outlined above the pion cross sections $\sigma_{\pi^{+}}\left(T_{p}, p^{i}\right)$ are available either as $\sigma_{\pi^{+}}\left(1.0 \mathrm{GeV}, p^{i}\right)$ (spectra from Ref. [14] downscaled in order to match the average cross section at $1.0 \mathrm{GeV}$ from Table 1 ) or as $\sigma_{\pi^{+}}\left(T_{p}, 500 \mathrm{MeV} / \mathrm{c}\right)$ (values given in Table 1 ). As a consequence, two different normalisation methods have to be used for the two data sets (1.3 and 1.6 $\mathrm{T}$ modes): the $1.3 \mathrm{~T}$ data comprise the measurement at $T_{p}=1.0 \mathrm{GeV}$ where the pion momentum spectrum is available in the full ANKE momentum range, whereas the 1.6 T data have been obtained at energies $T_{p} \geq 1.2 \mathrm{GeV}$ where only the cross sections at $p_{\pi^{+}}=500 \mathrm{MeV} / \mathrm{c}$ are known.

Normalisation of the $\mathbf{1 . 3} \mathbf{T}$ data: The pion and kaon data have been obtained at identical settings of the spectrometer, thus $(\Delta p \Delta \Omega)_{K^{+}}^{i}=(\Delta p \Delta \Omega)_{\pi^{+}}^{i}$. These angular-momentum bins can be calculated from Eq. (3):

$$
\begin{aligned}
(\Delta p \Delta \Omega)^{i} & =\frac{N_{\pi^{+}}^{i}\left(T_{p}\right)}{\sigma_{\pi^{+}}\left(T_{p}, p^{i}\right)} \cdot \frac{1}{\mathcal{L}_{\pi^{+}}\left(T_{p}\right)} \cdot \frac{1}{\epsilon_{\pi^{+}}^{i}} \\
& =\frac{N_{\pi^{+}}^{i}(1.0)}{\sigma_{\pi^{+}}\left(1.0, p^{i}\right)} \cdot \frac{1}{\mathcal{L}_{\pi^{+}}(1.0)} \cdot \frac{1}{\epsilon_{\pi^{+}}^{i}} .
\end{aligned}
$$

Using Eq. (2) one obtains:

$$
\begin{aligned}
\sigma_{K^{+}}\left(T_{p}, p^{i}\right)= & N_{K^{+}}^{i}\left(T_{p}\right) \cdot \\
& \frac{\sigma_{\pi^{+}}\left(1.0, p^{i}\right)}{N_{\pi^{+}}^{i}(1.0)} \cdot \frac{\mathcal{L}_{\pi^{+}}(1.0)}{\mathcal{L}_{K^{+}}\left(T_{p}\right)} \cdot \frac{\epsilon_{\pi^{+}}^{i}}{\epsilon_{K^{+}}^{i}} .
\end{aligned}
$$

The relative luminosity normalisation $\mathcal{L}_{\pi^{+}} / \mathcal{L}_{K^{+}}\left(T_{p}\right)$ has been obtained individually for each beam energy by monitoring the interaction of the proton beam with the target to an accuracy of $2 \%$ using stop counters $2-5$ in four-fold coincidence. This selects ejectiles which are produced in the target by hadronic interactions and bypass the spectrometer dipole D2, see Fig. 1. The signal of these monitors has been corrected for the dead time of the data acquisition system. For the more general case of different beam energies, i.e. for the ratio $\mathcal{L}_{\pi^{+}}(1.0) / \mathcal{L}_{K^{+}}\left(T_{p}\right)$ in Eq. (6), one has to use

$$
\begin{aligned}
\frac{\mathcal{L}_{\pi^{+}}(1.0)}{\mathcal{L}_{K^{+}}\left(T_{p}\right)} & \equiv \frac{\mathcal{L}_{\pi^{+}}}{\mathcal{L}_{K^{+}}}\left(T_{p}\right) \cdot \frac{\mathcal{L}_{\pi^{+}}(1.0)}{\mathcal{L}_{\pi^{+}}\left(T_{p}\right)} \\
& =\frac{\mathcal{L}_{\pi^{+}}}{\mathcal{L}_{K^{+}}}\left(T_{p}\right) \cdot \frac{\sigma_{\pi^{+}}\left(T_{p}, p^{i}\right)}{\sigma_{\pi^{+}}\left(1.0, p^{i}\right)} \cdot \frac{N_{\pi^{+}}^{i}(1.0)}{N_{\pi^{+}}^{i}\left(T_{p}\right)}
\end{aligned}
$$

Since Eq. (7) is valid for any telescope $i$ (i.e. momentum $p$ ) we choose $i=15$ since $\sigma_{\pi^{+}}\left(T_{p}, p^{i}\right)$ is only available for $p^{i=15}=500 \mathrm{MeV} / \mathrm{c}$ :

$$
\frac{\mathcal{L}_{\pi^{+}}(1.0)}{\mathcal{L}_{K^{+}}\left(T_{p}\right)}=\frac{\mathcal{L}_{\pi^{+}}}{\mathcal{L}_{K^{+}}}\left(T_{p}\right) \cdot \frac{\sigma_{\pi^{+}}\left(T_{p}, 500\right)}{\sigma_{\pi^{+}}(1.0,500)} \cdot \frac{N_{\pi^{+}}^{15}(1.0)}{N_{\pi^{+}}^{15}\left(T_{p}\right)} .
$$

Combining Eqs. (6) and (8) one finds the final formula for normalisation of the kaon cross sections:

$\sigma_{K^{+}}\left(T_{p}, p^{i}\right)=N_{K^{+}}^{i}\left(T_{p}\right)$

$$
\frac{\sigma_{\pi^{+}}\left(1.0, p^{i}\right)}{N_{\pi^{+}}^{i}(1.0)} \cdot \frac{\mathcal{L}_{\pi^{+}}}{\mathcal{L}_{K^{+}}}\left(T_{p}\right) \cdot \frac{\sigma_{\pi^{+}}\left(T_{p}, 500\right)}{\sigma_{\pi^{+}}(1.0,500)} \cdot \frac{N_{\pi^{+}}^{15}(1.0)}{N_{\pi^{+}}^{15}\left(T_{p}\right)} \cdot \frac{\epsilon_{\pi^{+}}^{i}}{\epsilon_{K^{+}}^{i}}
$$

Normalisation of the $\mathbf{1 . 6} \mathbf{T}$ data: For the higher field strength the spectra could not be measured at $T_{p}=$ $1.0 \mathrm{GeV}$, since this energy would result in a too large beam deflection angle in the ANKE dipoles [1]. Hence, Eq. (5) cannot be used and the $1.6 \mathrm{~T}$ data have been analysed applying identical cuts such that $(\Delta p \Delta \Omega)^{i}$ is constant (with an accuracy of better than 5\%) for all telescopes and beam energies. Emission angles of the analysed particles $\left|\theta_{\text {hor }}\right|<6^{\circ}$ and $\left|\vartheta_{\text {vert }}\right|<3.5^{\circ}$ have been selected, the momentum acceptance of each telescope has been restricted to $\Delta p<6 \mathrm{MeV} / \mathrm{c}$. In the $1.6 \mathrm{~T}$ mode particles with $p=500 \mathrm{MeV} / \mathrm{c}$ are detected in telescope 13, thus:

$$
\begin{aligned}
(\Delta p \Delta \Omega)^{i}= & (\Delta p \Delta \Omega)^{13}= \\
& \frac{N_{\pi^{+}}^{13}\left(T_{p}\right)}{\sigma_{\pi^{+}}\left(T_{p}, 500\right)} \cdot \frac{1}{\mathcal{L}_{\pi^{+}}\left(T_{p}\right)} \cdot \frac{1}{\epsilon_{\pi^{+}}^{13}} .
\end{aligned}
$$

Together with Eq. (2) this leads to:

$$
\begin{aligned}
\sigma_{K^{+}}\left(T_{p}, p_{i}\right)= & N_{K^{+}}^{i}\left(T_{p}\right) \cdot \\
& \frac{\sigma_{\pi^{+}}\left(T_{p}, 500\right)}{N_{\pi^{+}}^{13}\left(T_{p}\right)} \cdot \frac{\mathcal{L}_{\pi^{+}}}{\mathcal{L}_{K^{+}}}\left(T_{p}\right) \cdot \frac{\epsilon_{\pi^{+}}^{13}}{\epsilon_{K^{+}}^{i}} .
\end{aligned}
$$

In principle, the (simpler) $1.6 \mathrm{~T}$ normalisation formula could also be applied to the $1.3 \mathrm{~T}$ data. However, the latter were generally measured with lower statistics which would be further reduced by the restriction of the accepted angles $\Delta \Omega$ and momenta $\Delta p$ leading to significantly larger statistical uncertainties.

\subsection{Target-mass dependence of the cross sections}

The target-mass dependence of the $K^{+}$-production cross sections has been determined using the formula:

$$
R(A / \mathrm{C}) \equiv \frac{\sigma_{K+}^{A}}{\sigma_{K^{+}}^{\mathrm{C}}}\left(p^{i}\right)=\left[\frac{N_{K+}^{A}}{N_{K^{+}}^{\mathrm{C}}}\right]_{\text {tel. }(i)} \cdot \frac{\mathcal{L}^{\mathrm{C}}}{\mathcal{L}^{A}}
$$

Here the index " $A$ " stands for the copper, silver and gold targets, respectively, while "C" refers to carbon. $N_{K^{+}}$ is the number of identified kaons in each telescope $i$ after dead-time corrections. $\mathcal{L}$ stands for the integrated luminosity during data taking for the particular target.

In principle, absolute production cross sections could have been determined also for the heavier targets using the normalisation procedure described in the previous paragraph. However, absolute cross sections for $\mathrm{Cu}, \mathrm{Ag}$ and $\mathrm{Au}$ are not needed for the following analyses, but only the cross-section ratios $R(A / \mathrm{C})$. These have the advantage of higher accuracy since many possible systematic errors, such as corrections for the detection efficiencies in the ANKE range telescopes, cancel out. 
The luminosity ratio $\mathcal{L}^{\mathrm{C}} / \mathcal{L}^{A}$ in Eq. (11) can be deduced from the ratio of pion rates, measured under identical experimental conditions for each target during calibration runs using an on-line trigger optimised for $\pi^{+}$ detection:

$$
\frac{\mathcal{L}^{\mathrm{C}}}{\mathcal{L}^{A}}=\left[\frac{N_{\pi^{+}}^{\mathrm{C}}}{N_{\pi^{+}}^{A}}\right]_{\mathrm{tel} . \# 13 / 15} \cdot\left[\frac{\sigma_{\pi^{+}}^{A}}{\sigma_{\pi^{+}}^{\mathrm{C}}}\right]_{p=500 \mathrm{MeV} / \mathrm{c}} \cdot
$$

$N_{\pi^{+}}$is the number of pions detected by telescope \#13 (which detects ejectiles with momenta of $500 \mathrm{MeV} / \mathrm{c}$ in the $1.6 \mathrm{~T}$ mode) or \#15 (1.3 T mode), normalised to the proton-beam flux. For a momentum of $500 \mathrm{MeV} / \mathrm{c}$ the ratio of the pion-production cross sections $\sigma_{\pi^{+}}$can be extracted from experimental data $[14,15,18]$ taken in the energy range $T_{p}=0.73-4.2 \mathrm{GeV}$ at emission angles of $0^{\circ}, 2.5^{\circ}$ and $15^{\circ}$. Figure 3 shows the cross-section ratios for $\mathrm{Cu} / \mathrm{C}$ and $\mathrm{Pb} / \mathrm{C}$ calculated from those data.

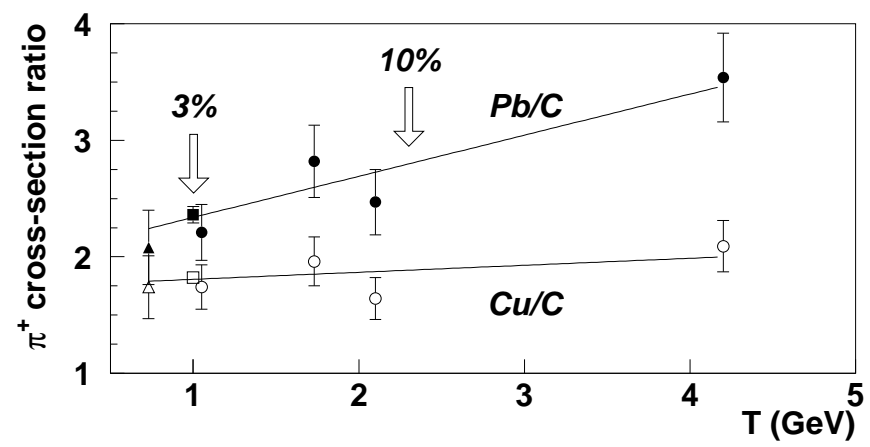

Fig. 3. Ratios of the $\pi^{+}$-production cross sections for $\mathrm{Cu} / \mathrm{C}$ and $\mathrm{Pb} / \mathrm{C}$ at $p_{\pi^{+}}=500 \mathrm{MeV} / \mathrm{c}$ as a function of the projectile energy $T_{p}$. The data were taken from Refs. [18] (triangles, $T_{p}=$ $0.73 \mathrm{GeV}, \vartheta_{\pi^{+}}=15^{\circ}$ ), [14] (squares, $T_{p}=1.0 \mathrm{GeV}, \vartheta_{\pi^{+}}=$ $0^{\circ}$ ) and [15] (circles, $T_{p}=1.05-4.2 \mathrm{GeV}, \vartheta_{\pi^{+}}=2.5^{\circ}$ ). The lines are linear fits to the data points. The arrows indicate the uncertainties of the ratios at the minimum and maximum energy measured with ANKE.

From Fig. 3 one can deduce the $\pi^{+}$-production ratios for $\mathrm{Cu} / \mathrm{C}$ and $\mathrm{Au} / \mathrm{C}$. For $\mathrm{Cu} / \mathrm{C}$ values of $1.81 \pm 0.05$ at $T_{p}=1.0 \mathrm{GeV}$ and $1.88 \pm 0.18$ at $T_{p}=2.3 \mathrm{GeV}$ are obtained. For $\mathrm{Pb} / \mathrm{C}$ the ratios are $2.34 \pm 0.07$ and $2.80 \pm 0.29$, respectively. All values agree within $10 \%$ with ratios scaling as $A^{1 / 3}$, i.e. 1.74 and 2.58 for $\mathrm{Cu} / \mathrm{C}$ and $\mathrm{Pb} / \mathrm{C}$, respectively. Thus, assuming an $A^{1 / 3}$ dependence, it is possible to calculate from the ratios $\mathrm{Pb} / \mathrm{C}$ those for $\mathrm{Au} / \mathrm{C}$. We use for the ratio $\mathrm{Au} / \mathrm{C}$ values of $2.29 \pm 0.07$ at $T_{p}=1.0 \mathrm{GeV}$ and $2.73 \pm 0.27$ at $T_{p}=2.3 \mathrm{GeV}$. The analysis of our $\pi^{+}$data shows that the cross-section ratios are to about $2 \%$ independent of the $\pi^{+}$-emission angle within the ANKE angular acceptance.

\section{Inclusive $K^{+}$-momentum spectra for} $A \geq 12$

\subsection{Absolute cross sections for $p^{12} \mathrm{C} \rightarrow K^{+} X$ reactions}

The measured cross sections for $K^{+}$production in $p \mathrm{C}$ interactions are shown in Fig. 4 and listed in Tables 4 and 5. The overall systematic uncertainties coming from the normalisation to pion data described in Sect. 2.2 (i.e. errors given in the right column of Table 1 plus $2 \%$ for the uncertainty of the beam monitoring) are $\epsilon_{\mathrm{syst}}=8,7,7,9$ and $10 \%$ for $T_{p}=1.0,1.2,1.5,2.0$ and $2.3 \mathrm{GeV}$, respectively. The error bars of the individual data points include the uncertainties arising from the efficiency-correction factors of Eq. (1) and (4) but not the overall uncertainty from the pion normalisation. In both operation modes of D2, kaons could be identified in telescopes \#3-15 whereas in the two closest telescopes the background from scattered particles turned out to be too large.

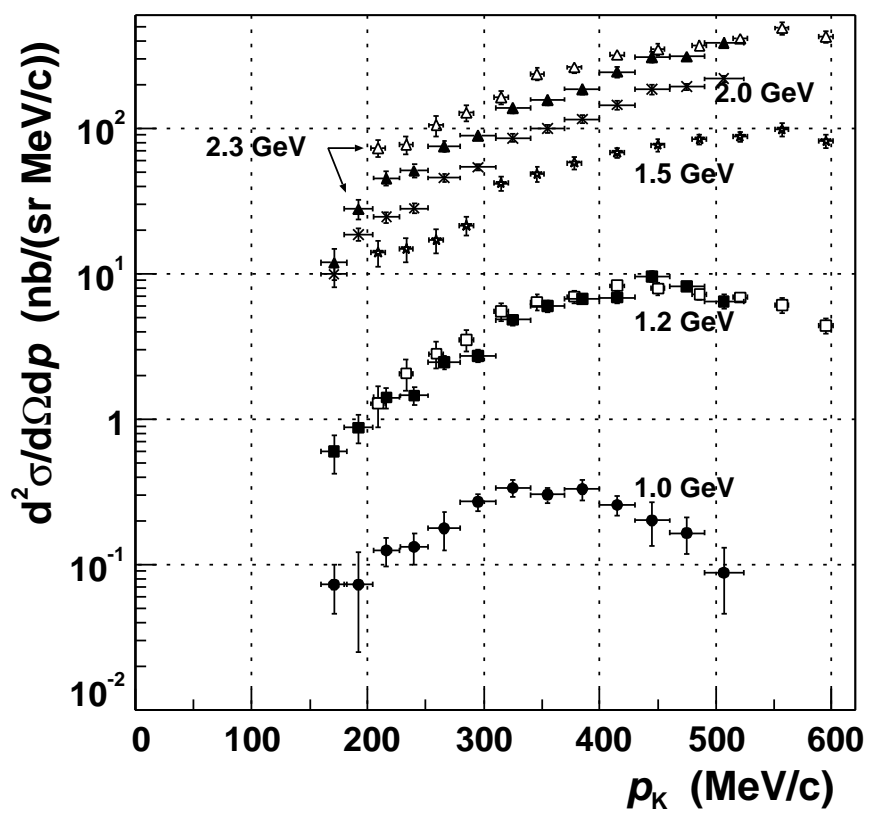

Fig. 4. Double differential $p^{12} \mathrm{C} \rightarrow K^{+} X$ cross sections measured at ANKE. The numerical values are reported in Tables 4 and 5 . Black circles denote the cross sections measured at $T_{p}=1.0 \mathrm{GeV}[2]$, squares at $T_{p}=1.2 \mathrm{GeV}$, stars at $T_{p}=1.5 \mathrm{GeV}$, crosses at $T_{p}=2.0 \mathrm{GeV}$ and triangles at $T_{p}=2.3 \mathrm{GeV}$. Closed symbols correspond to the measurements with $B=1.3 \mathrm{~T}$ in the full ANKE acceptance, while open symbols to $B=1.57 \mathrm{~T}$ and $\theta_{\text {hor }}<6^{\circ}$, $\vartheta_{\text {vert }}<3.5^{\circ}$. The error bars do not include the systematic uncertainties from the normalisation to pion data.

At $T_{p}=2.3 \mathrm{GeV}$ there are discrepancies between the two data sets for $p_{K^{+}} \leq 450 \mathrm{MeV} / \mathrm{c}$, whereas at $T_{p}=$ $1.2 \mathrm{GeV}$ they coincide. This cannot be caused by the angular dependence of $K^{+}$production because the cross sections evaluated in the even more restricted angular interval, $\left|\theta_{\text {hor }}\right|<4^{\circ}$ and $\left|\vartheta_{\text {vert }}\right|<2.5^{\circ}$ are in agreement with 
those obtained for $\left|\theta_{\text {hor }}\right|<6^{\circ}$ and $\left|\vartheta_{\text {vert }}\right|<3.5^{\circ}$. Furthermore, the discrepancy is unlikely to be due to the $K^{+}$identification procedure since, for this particular energy, different analysis algorithms were applied giving a difference in the cross sections smaller than $2 \%$. We conclude that there is an unknown source of systematic errors, which may yield an additional uncertainty of up to $30 \%$ at the highest measured beam energy. These uncertainties vanish when one calculates the cross-section ratios for different target nuclei, see Sect. 3.2.

\subsection{Target-mass dependence of $R(A / \mathrm{C})$}

The cross section ratios $R(A / \mathrm{C})$, measured with the two operation modes of $\mathrm{D} 2$, for beam energies $T_{p}=1.0,1.5$, 1.75 and $2.3 \mathrm{GeV}$ are listed in Tables $6,7,8$ and 9. These complement the published partial results $[2,3]$. No data for targets heavier than carbon was taken for $T_{p}=1.2$ and $2.0 \mathrm{GeV}$, whereas the absolute normalisation was not established at $T_{p}=1.75 \mathrm{GeV}$.

The measured ratios $R(\mathrm{Au} / \mathrm{C})$ at all beam energies are shown in Fig. 5. For $T_{p} \geq 1.5 \mathrm{GeV}$ all data exhibit similar shapes, rising steadily with decreasing kaon momenta, passing a maximum and falling steeply at low momenta. The maxima around $p_{\max } \sim 245 \mathrm{MeV} / \mathrm{c}$ coincide within $2 \mathrm{MeV} / \mathrm{c}$, i.e. their positions do not depend on the proton beam energy. The independence of the peak positions from the beam energy $T_{p}$ suggests that the effect is due to FSI effects of the produced kaons, since the basic production mechanism may change from 1.5 to $2.3 \mathrm{GeV}$ (see Sect. 4). The ratio data at $T_{p}=1.0 \mathrm{GeV}$ is much less precise and does not extend below $p_{K} \sim 220 \mathrm{MeV} / \mathrm{c}$ because of the large background at this low beam energy. Therefore, it is inconclusive with regard to the peak shape and position observed at the higher energies.

To ensure that the pronounced peak structure of $R(A / \mathrm{C})$ is not an artefact of the ANKE detection system, the $2.3 \mathrm{GeV}$ data were taken with both operation modes of $\mathrm{D} 2$, resulting in a change in the values of the momenta that are focused onto the individual range telescopes [12]. The data from Table 9 for $R(A / \mathrm{C})$ at $T_{p}=2.3 \mathrm{GeV}$ obtained with the $1.3 \mathrm{~T}$ and $1.6 \mathrm{~T}$ modes, show that these data sets agree, as illustrated in Fig. 6 for $R(\mathrm{Au} / \mathrm{C})$. This also demonstrates that the $30 \%$ uncertainty of the absolute cross sections measured at $T_{p}=2.3 \mathrm{GeV}$ cancels out for the ratios. In addition, the statistical errors are significantly smaller here. Consistent values for the ratios were also obtained when the polar $K^{+}$-emission angles were restricted to $\vartheta_{K^{+}}<3^{\circ}$, as shown in Fig. 6 .

The ratios of kaon-production cross sections $R(\mathrm{Cu} / \mathrm{C})$, $R(\mathrm{Ag} / \mathrm{C})$ and $R(\mathrm{Au} / \mathrm{C})$ measured at $2.3 \mathrm{GeV}$ are presented in Fig. 7. The position of the maximum of $R(\mathrm{~A} / \mathrm{C})$ varies with nucleus; a fit to the data results in $p_{\max }=$ $211 \pm 6,232 \pm 6$ and $245 \pm 5 \mathrm{MeV} / \mathrm{c}$ for $\mathrm{Cu}, \mathrm{Ag}$ and $\mathrm{Au}$, respectively [3].

In an earlier publication [3] we showed that from the cross section ratios $R(\mathrm{~A} / \mathrm{C})$ the nuclear $K^{+}$-potential for normal nuclear density can be derived with high accuracy. This is illustrated in Fig. 7, where the results of

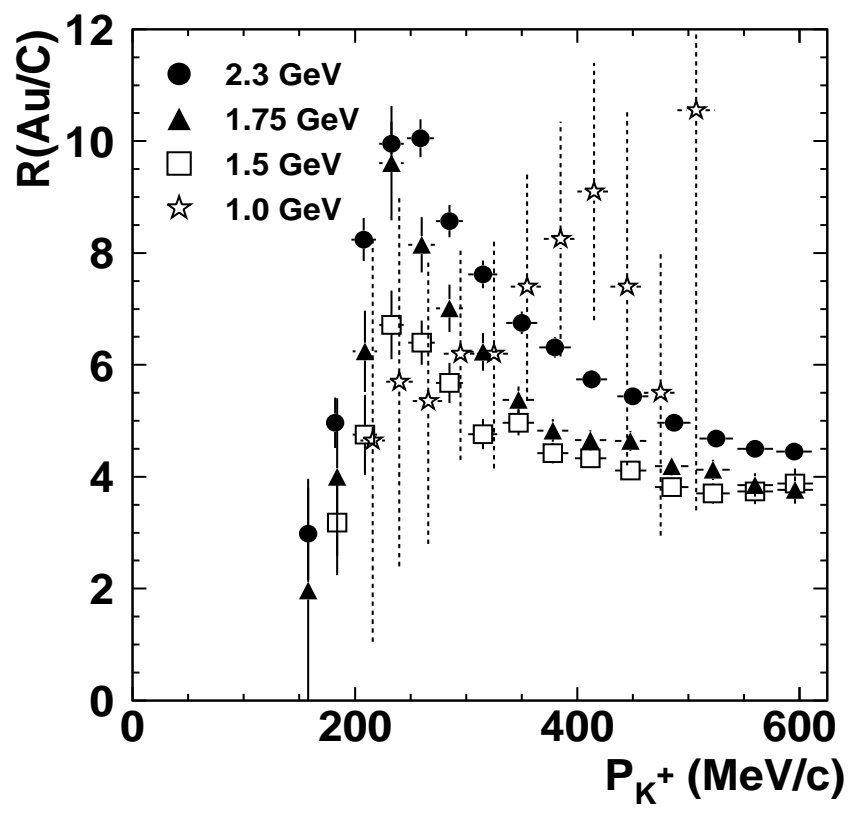

Fig. 5. Ratios of the $K^{+}$-production cross sections $R(\mathrm{Au} / \mathrm{C})$ measured at $T_{p}=1.0,1.5,1.75$ and $2.3 \mathrm{GeV}$ as a function of the laboratory kaon momentum $p_{K^{+}}$(cf. Tables $\left.6-9\right)$.

model calculations for $R(\mathrm{Au} / \mathrm{C})$ within the CBUU transport code $[4,5]$ are plotted. Without any FSI the ratio $R(\mathrm{Au} / \mathrm{C})$ is almost independent of the kaon momentum, and the average ratio $R(\mathrm{Au} / \mathrm{C}) \sim 9$ corresponds to an $A$-dependence $\sigma \propto A^{\alpha}$ with $\alpha \sim 0.79$.

It has frequently been argued that the $A$-dependence of the $K^{+}$-production cross section is sensitive to the dominant production mechanism. In a naive model for direct $K^{+}$-production in a collision of the beam proton with a single target nucleon the $A$-dependence is given by the inelastic scattering cross section of the incident proton which can be parameterised as $A^{\alpha}$ with $\alpha \sim 0.6-0.7$ (see Ref. [19] and references therein). Nuclear effects, like the intrinsic momenta of the participating nucleons or Pauli blocking should not depend on the target mass. Therefore, one expects that the $K^{+}$-production cross section is proportional to $\sim A^{0.6-0.7}$ if the kaons are dominantly produced via the direct mechanism. $K^{+}$-mesons can also be produced in two-step processes with intermediate pion production $\left(p N_{1} \rightarrow \pi X\right)$ and subsequent $\pi N_{2} \rightarrow K^{+} X$ reactions on a second target nucleon $[5,13,20-24]$. Since two nucleons are needed in this case, a stronger $A$-dependence with $\alpha \gtrsim 1$ is expected. However, the analysis of $K^{+}$production data in Ref. [19] shows that it is not possible to draw unambiguous conclusions about the underlying reaction mechanisms from the $A$-dependence of differential $K^{+}$cross sections. The reason for this is that FSI effects change the shape of the kaon spectra and, as a result, the $A$-dependence may vary with the kaon momenta and emission angles covered by the particular experiment. For example, rescattering processes tend to slow down the produced kaons leading to a steadily rising ratio $R(\mathrm{Au} / \mathrm{C})$ with decreasing $K^{+}$momenta, see dashed line in Fig. 7 . 


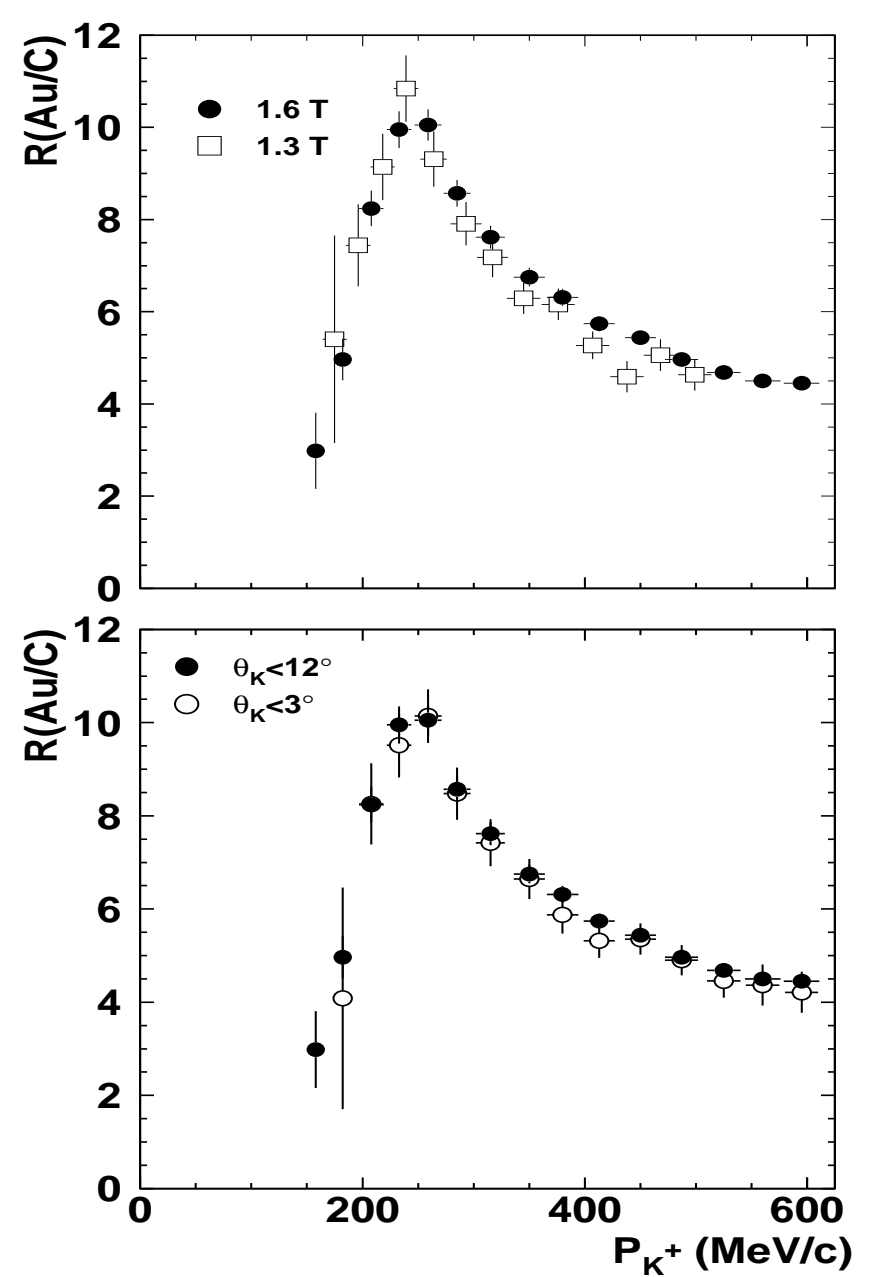

Fig. 6. Upper: Ratios of the $K^{+}$-production cross sections $R(\mathrm{Au} / \mathrm{C})$ measured at $T_{p}=2.3 \mathrm{GeV}$ and different operation modes of D2 (cf. Table 9). Lower: Same ratios measured in the full ANKE acceptance and restricted to angles $\vartheta_{K^{+}}<3^{\circ}$.

In a purely classical picture, kaons produced at some radius $R$ in a nucleus acquire an additional momentum of $p_{\min }=\sqrt{2 m_{K} V_{C}(R)}$ in the repulsive Coulomb potential. This corresponds to a minimum momentum of $p_{K} \sim$ $130 \mathrm{MeV} / \mathrm{c}$ for an Au nucleus. Thus $R(\mathrm{Au} / \mathrm{C})$ should drop to zero for smaller kaon momenta. This is well reproduced by the calculations presented in Fig. 7 and results in the pronounced peak structure of the cross-section ratios. From this peak position, which can be determined with high accuracy both in the data and the calculated spectra, the nuclear $K^{+}$-potential can be determined with high accuracy [3].

The repulsive nuclear $K^{+}$-potential, which is expected to be of strength $V_{K}=22 \pm 5 \mathrm{MeV}$ [25], independent of the target mass $A$, shifts the kaon momenta, and thus the peak positions, to higher values. In Fig. 7 we illustrate this effect for $V_{K}=0,20$ and $40 \mathrm{MeV}$. The best agreement between the measured and calculated peak positions is obtained for $V_{K}=20 \mathrm{MeV}$ with an estimated uncertainty of about $\pm 3 \mathrm{MeV}$. We expect that an even higher precision in $V_{K}$
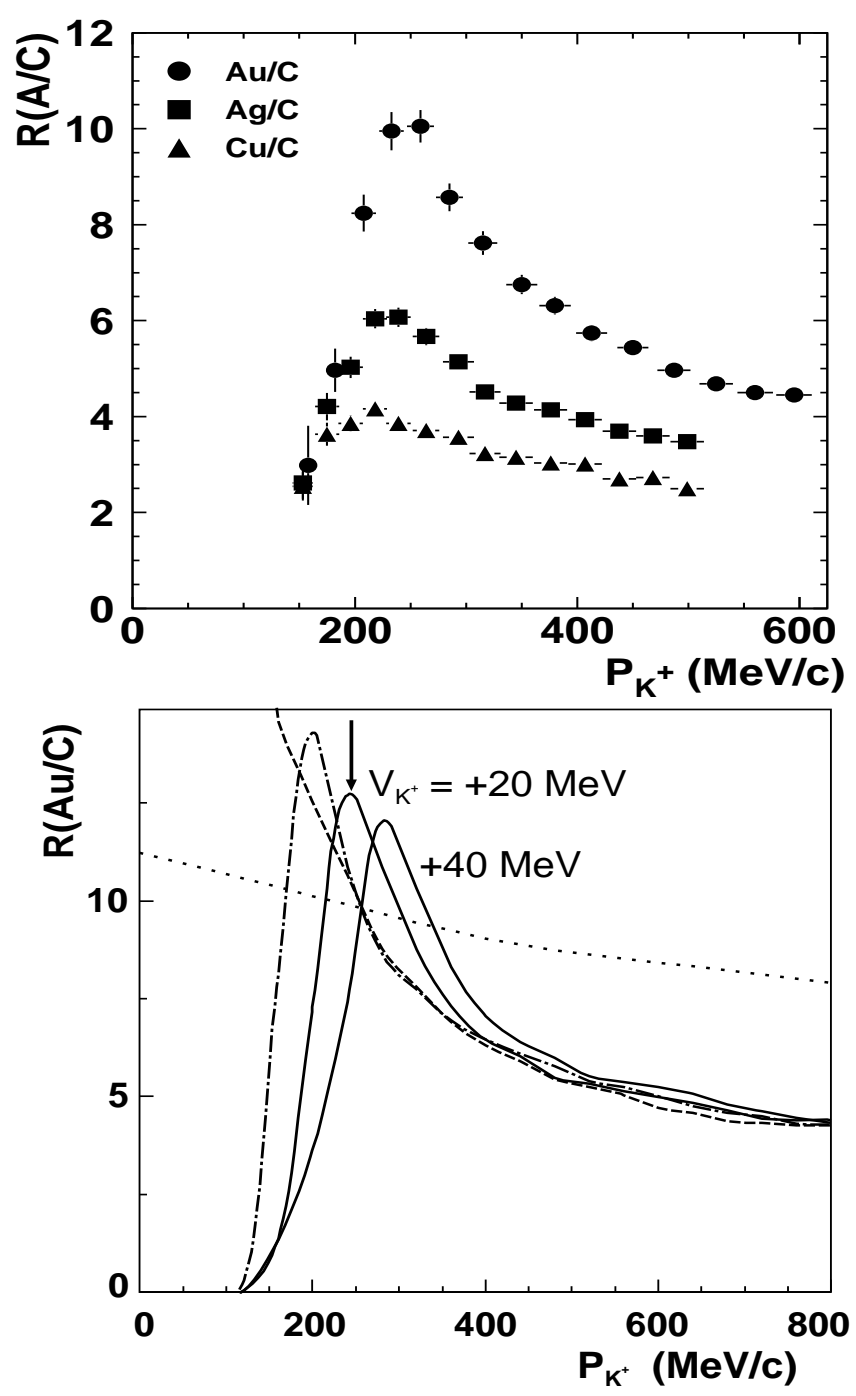

Fig. 7. Upper: Ratios of the $K^{+}$-production cross sections $R(\mathrm{Cu} / \mathrm{C})(1.3 \mathrm{~T}), R(\mathrm{Ag} / \mathrm{C})(1.3 \mathrm{~T}), R(\mathrm{Au} / \mathrm{C})(1.6 \mathrm{~T})$ measured at $T_{p}=2.3 \mathrm{GeV}$ as a function of the laboratory kaon momentum (cf. Table 9). Lower: Result of model calculations with the CBUU transport code [5] for $R(\mathrm{Au} / \mathrm{C})$ showing the effect of $K^{+}$rescattering, the Coulomb and nuclear $K^{+}$potentials (dotted line: no rescattering and no potentials; dashed: only rescattering, no potentials; dashed-dotted: rescattering plus Coulomb; solid: full calculation taking into account rescattering, Coulomb and different nuclear potentials). The arrow indicates the position of the maximum of the measured ratio $R(\mathrm{Au} / \mathrm{C})$.

could be obtained from refined model calculations for the full data set (all beam energies and target nuclei) which not only reproduce the peak positions of $R(\mathrm{~A} / \mathrm{C})$ but also the shape of all measured distributions, in particular at low kaon momenta [26]. 


\section{Systematics of available $p \mathrm{C}$ cross sections}

Table 2 lists the available data on $K^{+}$production in $p A$ collisions from ANKE and elsewhere in the literature. The different data sets were obtained for non-overlapping kinematical parameters (i.e. beam energies, kaon emission angles and momenta) which prevents a direct comparison with the results from ANKE and, e.g., a test of the overall normalisations.

In Ref. [19] it was shown that the measured invariant cross sections $E \mathrm{~d}^{3} \sigma / \mathrm{d}^{3} p$ follow an exponential scaling behaviour when plotted as a function of the four-momentum transfer $t$, as illustrated in Fig. 8.

Apart from the data taken with ANKE at $T_{p}=1.0$ and $1.2 \mathrm{GeV}$, all spectra cover the range of negative $t$. The sharp fall-off of the cross sections from ANKE towards positive values of $t$ was explained in Ref. [19] by the fact that the data were taken very close to the kinematical limit for hypernucleus formation at $t=0.145 \mathrm{GeV}^{2}\left(T_{p}=\right.$ $1.0 \mathrm{GeV}$ ) which is usually accompanied by very small cross sections. For $t<0$ the individual data sets $i$ show exponential dependences of the form $E \mathrm{~d}^{3} \sigma / \mathrm{d}^{3} p=c_{0}^{i} \exp \left[b_{0}^{i} t\right]$, with parameters $c_{0}^{i}$ and $b_{0}^{i}$ being given in Ref. [19]. It has been speculated in Ref. [19] that the differing slope parameters $b_{0}^{i}$, as well as deviations from the exponential behaviour (see, e.g., the data from KaoS [32] in Fig. 8), reflect a dependence on the available squared CM energy $s$ and the excitation energy of the target nucleus, $\Delta m=m_{X}-m_{A}$, where $m_{X}$ and $m_{A}$ denote the masses of the target nucleus before and after the reaction process, respectively. Based on Regge phenomenology [33] the following formula has been suggested as a parameterisation of the invariant cross section:

$$
E \frac{\mathrm{d}^{3} \sigma}{\mathrm{d}^{3} p} \propto f\left(t, m_{X}^{2}\right) \exp \left[b_{0} t \cdot \ln \left(s / s_{0}\right)\right] .
$$

It is shown in the following that an explicit treatment of the $s$ and $\Delta m$ dependences based on Eq. (13) allows one to describe all data sets with a single parameter $b_{0}$, expressing the average $t$ dependence of the invariant $K^{+}$production cross section in $p A$ interactions (see dashed line in Fig. 8 which has been calculated for an arbitrary value of $c_{0}$ ).

Figure 8 shows the invariant cross sections, divided by the factor $\exp \left[b_{0} t \cdot \ln \left(s / s_{0}\right)\right]$ using $b_{0}=2.2 \mathrm{GeV}^{-2}$ and $s_{0}=100 \mathrm{GeV}^{2}$, as a function of the excitation energy $\Delta m$. The two solid lines correspond to a parameterisation of the invariant cross sections in $p^{12} \mathrm{C}$ interactions

$$
\begin{aligned}
E \frac{\mathrm{d}^{3} \sigma}{\mathrm{d}^{3} p} & =\sigma_{0} \cdot \Delta m^{N_{0}} \cdot \exp \left[b_{0} t \cdot \ln \left(s / s_{0}\right)\right] \\
& =\sigma_{0} \cdot \Delta m^{N_{0}} \cdot\left(s / s_{0}\right)^{b_{0} t}
\end{aligned}
$$

with fitted parameters as summarised in Table 3 . The term $\Delta m^{N_{0}}$ reflects a phase-space behaviour $\sigma \propto \Delta m^{N_{0}}=$ $\Delta m^{(3 n-5) / 2}$ with $n$ particles in the final state.

As can be seen from Fig. 8, the parameterisation from Eq. (14) can describe all available data on $K^{+}$production in $p A$ reactions, obtained at various detection angles and
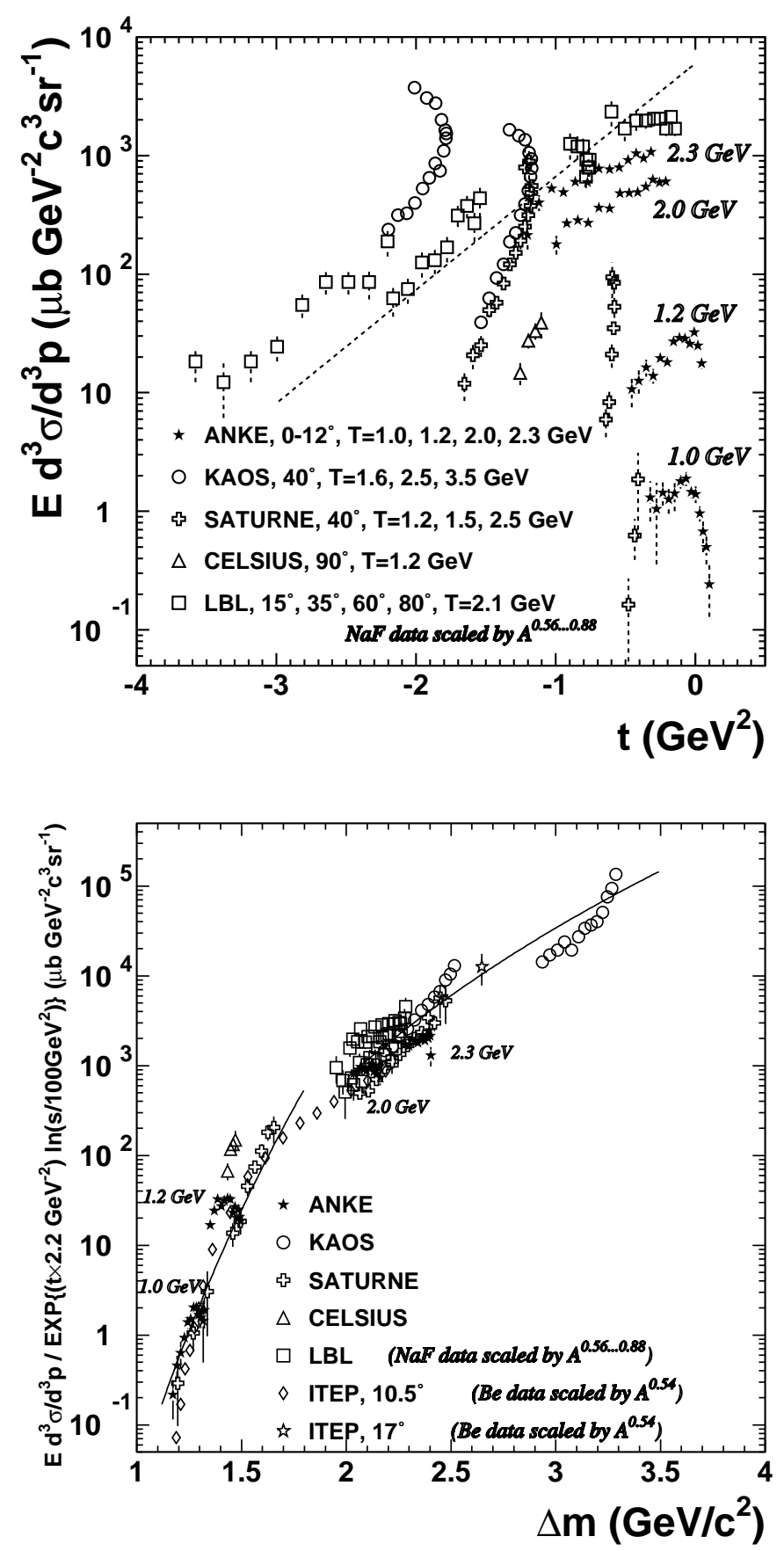

Fig. 8. Upper: Invariant $p^{12} \mathrm{C} \rightarrow K^{+} X$ cross sections as a function of the four-momentum transfer $t$ between the beam proton and the outgoing kaon. The dashed line indicates the average $t$ dependence, obtained from a global fit to all data sets using Eq. (14) $\left(b_{0}=2.2 \mathrm{GeV}^{-2}\right)$. Lower: Scaled invariant $p^{12} \mathrm{C} \rightarrow K^{+} X$ cross sections as a function of the excitation energy $\Delta m$ of the target nucleus. The data from LBL [27] and ITEP $[30,31]$ were measured with $\mathrm{NaF}(\mathrm{Be})$ targets and were corrected for the target-mass dependence according to Ref. [19]. The beam energies of the ANKE data sets (filled stars) are indicated; for $T_{p}=2.3 \mathrm{GeV}$ the data obtained with $B=1.3 \mathrm{~T}$ (closed triangles in Fig. 4) were used. 
Table 2. Data on $K^{+}$-production in $p A$ collisions (ordered by the year of publication) at various beam energies $T_{p}$, kaon momenta $p_{K}$ and emission angles $\vartheta_{K}$.

\begin{tabular}{ccccc}
\hline$T_{p}(\mathrm{GeV})$ & Targets & $p_{K}(\mathrm{MeV} / \mathrm{c})$ & $\vartheta_{K}\left(^{\circ}\right)$ & Measured at \\
\hline $0.842-0.99$ & $\mathrm{Be} . \mathrm{Pb}$ & total cross & sections & PNPI [13] \\
2.1 & $\mathrm{NaF}, \mathrm{Pb}$ & $350-750$ & $15-80$ & LBL [27] \\
$1.2,1.5,2.5$ & $\mathrm{C}, \mathrm{Pb}$ & $500-700$ & 40 & SATURNE [28] \\
1.2 & $\mathrm{C}$ & $165-255$ & 90 & CELSIUS [29] \\
$1.65-2.91$ & $\mathrm{Be}, \mathrm{Al}, \mathrm{Cu}, \mathrm{Ta}$ & 1280 & 10.5 & ITEP [30] \\
2.9 & $\mathrm{Be}$ & 545 & 17 & ITEP [31] \\
1.0 & $\mathrm{C}, \mathrm{Cu}, \mathrm{Au}$ & $171-507$ & $\leq 12$ & ANKE [2] \\
1.6 & $\mathrm{C}, \mathrm{Au}$ & $275-1075$ & $40-64$ & KaoS [32] \\
1.6 & $\mathrm{Ni}$ & $275-1075$ & 40 & KaoS [32] \\
$2.5,3.5$ & $\mathrm{C}, \mathrm{Au}$ & $275-1075$ & $32-64$ & KaoS [32] \\
$2.5,3.5$ & $\mathrm{Ni}$ & $275-1075$ & 40 & KaoS [32] \\
$1.0,1.2,1.5,2.0,2.3$ & $\mathrm{C}, \mathrm{Cu}, \mathrm{Ag}, \mathrm{Au}$ & $171-595$ & $\leq 12$ & ANKE \\
\hline
\end{tabular}

in different momentum domains, within a factor $\sim 2-3$. Within this uncertainty the cross sections from ANKE are in agreement with those from other experiments. Furthermore, the parameterisation given by Eq. (14) supplies a Lorentz-invariant description of all available data which is independent of the choice of the reference system, $p p$ or $p A$.

Table 3. Parameters obtained from a fit with Eq. (14).

\begin{tabular}{ccccc}
\hline $\begin{array}{c}T_{p} \\
(\mathrm{GeV})\end{array}$ & $\begin{array}{c}\sigma_{0} \\
\left(\mathrm{nb} \mathrm{GeV}^{-2} \mathrm{c}^{3} \mathrm{sr}^{-1}\right)\end{array}$ & $N_{0}(n)$ & $\begin{array}{c}b_{0} \\
\left(\mathrm{GeV}^{-2}\right)\end{array}$ & $\begin{array}{c}s_{0} \\
\left(\mathrm{GeV}^{2}\right)\end{array}$ \\
\hline$<1.58$ & 25 & $17(13)$ & & 100 \\
$>1.58$ & 1000 & $9.5(8)$ & & \\
\hline
\end{tabular}

The fitted parameters together with Fig. 8 reveal the following kinematical features of $K^{+}$-production processes in nuclei:

- The kinematical limit for the production of $K^{+}$-mesons in a $p A$ reaction is at $\Delta m_{\min }=m_{\Lambda}=1.116 \mathrm{GeV} / \mathrm{c}^{2}$. In this limiting case only a $\Lambda$-Hyperon is produced and no energy can be transferred to excite the target nucleus or knock out target nucleons. The initial target nucleus must take part in the reaction as a whole such that the effective target mass is $12 \cdot m_{N}$. There are only two particles, $K^{+}$-meson and hypernucleus, in the final state (corresponding to $n=2$ ).

- For excitation energies $\Delta m$ between $1.173 \mathrm{GeV} / \mathrm{c}^{2}$ (i.e. data point from ANKE at $T_{p}=1.0 \mathrm{GeV}$ and $p_{K^{+}}=$ $507 \mathrm{MeV} / \mathrm{c}$ ) and $\sim 1.7 \mathrm{GeV} / \mathrm{c}^{2}$ (beam energies below $T_{N N}=1.58 \mathrm{GeV}$ ) the production cross sections steeply rise. Within the phase-space treatment of the data $\left(N_{0}=17, n=13\right)$ this indicates that all $12 \mathrm{nu}-$ cleons plus the $\Lambda$-hyperon should carry away energy in the final state.
- At higher excitation energies $\Delta m \gtrsim 2.0 \mathrm{GeV} / \mathrm{c}^{2}\left(T_{p}>\right.$ $1.58 \mathrm{GeV})$ the rise of the cross sections becomes less steep $\left(N_{0}=9.5, n=8\right)$.

These kinematical features can be linked to the mechanisms leading to $K^{+}$production in $p A$ interactions:

1. The $1.0 \mathrm{GeV}$ ANKE data were obtained very close to the kinematical limit at $\Delta m_{\min }=m_{\Lambda}=1.116 \mathrm{GeV} / \mathrm{c}^{2}$. If the measurements were extended to even larger kaon momenta or to smaller beam energies (i.e. closer to the kinematical limit) the steep drop of the cross sections $(n=13)$ would end in the regime of hypernucleus production where $n=2$ is expected.

2. The simple phase-space description of the final state with $n=13$ indicates that below threshold the $K^{+}$production mechanisms have to transfer energy to all 12 target nucleons and the produced hyperon. However, this approach neglects collective effects in the initial target nucleus. Thus the steep drop of the cross sections might also reflect the fact that more and more nucleons must take part in the kaon-production process when approaching the kinematical limit (up to $\left.12 \cdot m_{N}\right)$. In fact, the ANKE data at $1.0 \mathrm{GeV}$ reveal a high degree of collectivity in the target nucleus (see Ref. [2]). For example, if the kaons are produced in a collision of the $1.0 \mathrm{GeV}$ beam proton with a single target nucleon, internal momenta of at least $\sim 550 \mathrm{MeV} / \mathrm{c}$ are needed in order to produce kaons in the forward direction with momenta of $p_{K^{+}} \sim 500 \mathrm{MeV} / \mathrm{c}$. Such high momentum components are essentially due to manybody correlations in the nucleus. Within a very simple phase-space approximation (which neglects the intrinsic motion of the target nucleons) it can be shown that the number of participating target nucleons must be $\sim 5-6[2]$. It has been suggested [5,13,20-24] that such effects can be described in terms of multi-step mechanisms or high-momentum components in the nuclear wave function.

3. The different slope parameters below and above the free $N N$ threshold indicate a change of the $K^{+}$-production mechanism. This has been proposed long ago 
in Ref. [13] and is confirmed by microscopical model calculations [5,20-24] and recent $K^{+} d$ coincidence measurements with ANKE [34]. These suggest that below threshold the kaons are dominantly produced in two-step reactions with intermediate pion formation, whereas at higher energies direct $K^{+}$production on a single nucleon prevails.

\section{Inclusive $K^{+}$cross sections from $p \mathrm{D}$ interactions}

$K^{+}$-production in $p \mathrm{D}$ interactions was measured at two beam energies, $T_{p}=1.83$ and $2.02 \mathrm{GeV}$. ANKE was operated in the $1.45 \mathrm{~T}$ and $1.6 \mathrm{~T}$ modes and the vertical and horizontal kaon detection angles were restricted to $\vartheta<4^{\circ}$. For absolute normalisation of the cross sections the luminosity $\mathcal{L}$ has been determined with the help of events from $p d$ elastic scattering. These have been measured with the ANKE spectator-detection system; details of the detectors and the normalisation procedure are described in Ref. [11]. The overall systematic uncertainty in the absolute normalisation is $20 \%$. All other analysis procedures are in accord with those for the heavier targets described in Sect. 2.1.

Figure 9 shows the $K^{+}$-momentum spectra measured at beam energies $T_{p}=1.83 \mathrm{GeV}$ and $2.02 \mathrm{GeV}$ on the deuteron target and at $2.0 \mathrm{GeV}$ on carbon. The difference between the $p \mathrm{D}$ cross sections can be attributed to the fact that the lower energy is significantly closer to the free nucleon-nucleon threshold. The different $p \mathrm{D}$ and $p \mathrm{C}$ cross sections at $T_{p}=2.0 \mathrm{GeV}$ can be explained in the framework of the Glauber model [35]. In this model, beam protons effectively "sees" 7.3 nucleons in a carbon target $[5,13]$. Thus the ratio of the $p \mathrm{C}$ and $p \mathrm{D}$ cross sections should be about 3.5, which is confirmed by the data. This finding suggests that at $T_{p}=2.0 \mathrm{GeV}$ kaons are produced on individual nucleons both in deuteron and carbon targets. There is no indication for any collective target behaviour. Furthermore, shadowing effects in the deuteron are smaller than 5\% [36]. Therefore, for the following analysis, which is carried out in a simple phase-space approach, we assume $\sigma_{D}=\sigma_{p}+\sigma_{n}$, with $\sigma_{n} / \sigma_{p}$ being a free parameter.

Following the analysis in Ref. [7] of different inclusive reactions giving rise to $K^{+}$-production, six main reaction channels lead to kaon production on the deuteron at our beam energies. They are listed in Table 11 together with the corresponding cross sections which have been calculated using the parameterisations given in Ref. [7]. The calculated value for $\sigma\left(p p \rightarrow p K^{+} \Lambda\right)$ at $2.0 \mathrm{GeV}$ is in agreement with the experimental result from Ref. [37]. For the simulation of phase-space distributed $p \mathrm{D} \rightarrow K^{+} X$ events, the PLUTO package [38], which takes into account the intrinsic motion of the nucleons in the deuteron, has been used. The events have been generated for each reaction channel and weighted according to the total cross sections from Table 11. Each event subsequently has been tracked through the spectrometer dipole and all detection

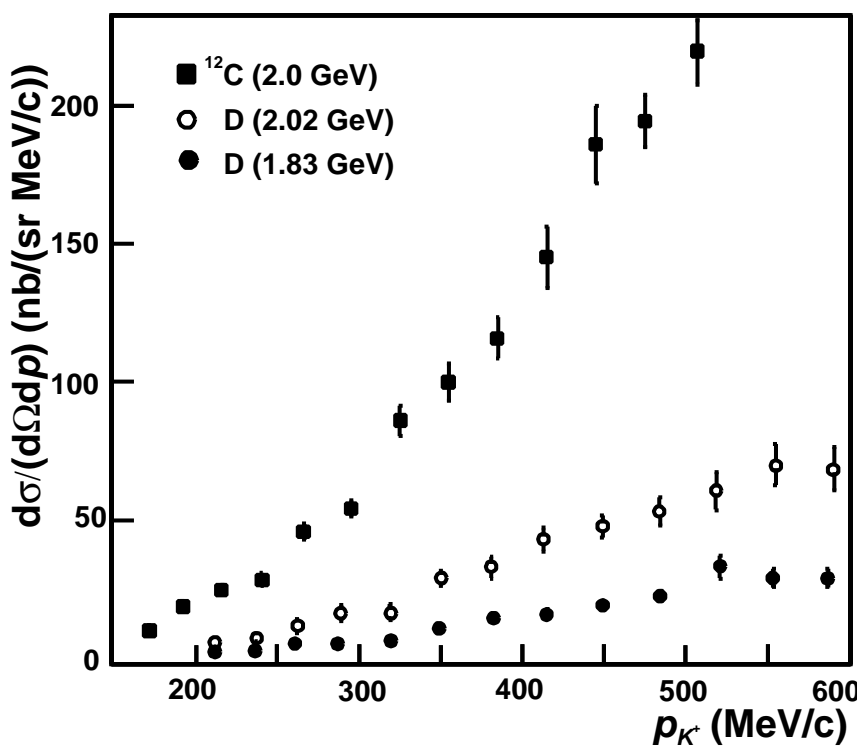

Fig. 9. Double differential cross sections for $K^{+}$production on a deuteron target measured at $T_{p}=1.83$ and $2.02 \mathrm{GeV}$ (c.f. Table 10) and on a carbon target at $2.0 \mathrm{GeV}$ (c.f. Table 4).

efficiencies have been taken into account. A comparison of the simulated spectra with the data is shown in Fig. 10 (curve labelled by " $\sigma_{n}=2 \sigma_{p}$ ").
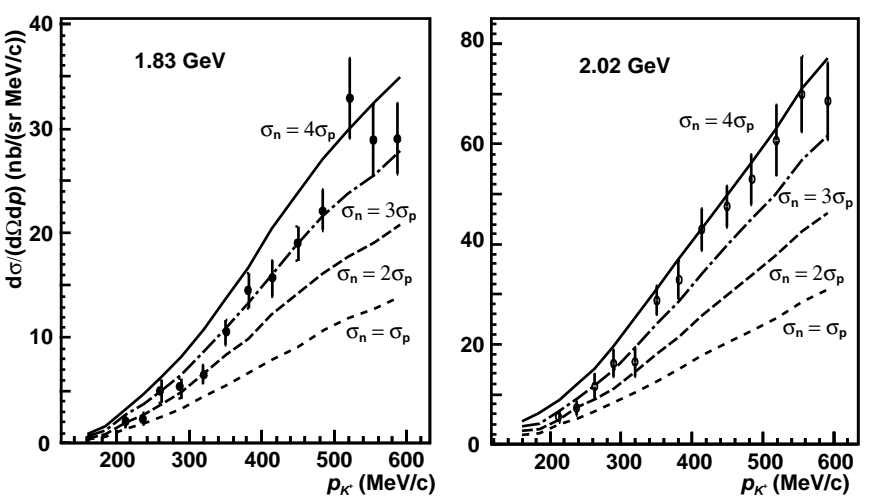

Fig. 10. Double differential $p \mathrm{D} \rightarrow K^{+} X$ cross section at $1.83 \mathrm{GeV}$ and at $2.02 \mathrm{GeV}$ in comparison with our model calculations using different ratios $\sigma_{n} / \sigma_{p}$ (lines).

The apparent difference between the calculated and measured cross sections can be due to the following reasons: our simple approach does not take into account the dynamics of the interaction in the different states (such as initial- and final-state interactions) and the ratio $\sigma_{n} / \sigma_{p}$ might be different from that suggested in Ref. [7]. Thus we repeated the simulations keeping the relative weights of the three $p p$ and $p n$ channels constant (as given in Table 11) while treating the ratio of the sum of these two contributions, i.e. $\sigma_{n} / \sigma_{p}$, as a free parameter. The result of the simulations with different ratios $\sigma_{n} / \sigma_{p}$ is also shown in Fig. 10. The best agreement between data and calcula- 
tions is obtained for $\sigma_{n} / \sigma_{p} \sim 3$ at $1.83 \mathrm{GeV}$ and $\sigma_{n} / \sigma_{p} \sim 4$ at $2.02 \mathrm{GeV}$.

\section{Summary and outlook}

In this paper we present the full set of inclusive data on $p A \rightarrow K^{+} X$ reactions measured at forward angles with the ANKE spectrometer at COSY-Jülich. Far below the free nucleon-nucleon threshold there must be a strong collective behaviour of the target nuclei, whereas a change of the reaction mechanism is observed at higher energies. For a better understanding of the reaction mechanisms further microscopical model calculations should be performed. Such calculations are also needed to extract the precise value of the in-medium nuclear $K^{+}$-potential from the cross-section ratios $R(A / \mathrm{C})$ measured at ANKE.

The inclusive $K^{+}$cross sections from ANKE are compatible with those obtained at other facilities. All data sets together now cover a wide kinematical range, i.e. beam energies, kaon momenta and emission angles. We currently do not foresee further inclusive measurements since we regard the existing data sets - in particular with recent results from the KaoS collaboration obtained at larger kaon-emission angles [32] — as being largely sufficient. However, we expect that significant progress for the understanding of the $\mathrm{K}^{+}$-production mechanisms will come from correlation data, where the produced kaons are measured in coincidence with protons, deuterons and other light fragments. A first step in this direction has already been made at ANKE. Data on the reaction $p \mathrm{C} \rightarrow K^{+} d X$ at $1.2 \mathrm{GeV}$ provide the first direct indication for kaon production in the particular two-step reaction $p N_{1} \rightarrow \pi d$, $\pi N_{2} \rightarrow K^{+} \Lambda$ with formation of intermediate pions in the target nucleus [34]. The data also hint on processes involving $p(2 N) \rightarrow K^{+} d \Lambda$ reactions on two-nucleon clusters.

First correlation measurements have also been performed using deuteron targets. A preliminary analysis of the proton-momentum spectra from $p \mathrm{D} \rightarrow K^{+} p X$ events confirm our present conclusion from the inclusive data that the $K^{+}$-production cross section on neutrons is significantly larger than on protons [39]. This finding should be taken into account in future model calculations, especially of $K^{+}$production in heavy ion reactions.

It is also planned to measure at ANKE the production of $K^{+} K^{-}$pairs in $p A$ collisions. It can be expected that the measurement of low-momentum kaons offers the unique possibility to determine the nuclear $K^{-}$-potential for normal nuclear density with similar accuracy to that of the $K^{+}$-mesons.

\section{Acknowledgements}

This work profitted significantly from discussions with members of the ANKE collaboration, in particular W. Cassing, A. Sibirtsev and C. Wilkin. We are also grateful to R. Schleichert as the technical coordinator of ANKE and to C. Wilkin for carefully reading the manuscript. Financial support from the following funding agencies was of indispensable help for building ANKE, its detectors and DAQ: Germany (BMBF: grants WTZ-RUS-649-96, WTZ-RUS-666-97, WTZ-RUS-685-99, WTZ-POL-007-99, WTZ-POL-015-01, WTZ-POL-041-01; DFG: 436 RUS 113/ 337, 436 RUS 113/444, 436 RUS 113/561, State of NorthRhine Westfalia), Poland (Polish State Committee for Scientific Research: 2 P03B 101 19), Russia (Russian Ministry of Science: FNP-125.03, Russian Academy of Science: 99-02-04034, 99-02-18179a) and European Community (INTAS-98-500).

\section{References}

1. S. Barsov et al., Nucl. Instr. Methods Phys. Res., Sect. A 462, 364 (2001).

2. V. Koptev et al., Phys. Rev. Lett. 87, 022301 (2001).

3. M. Nekipelov et al., Phys. Lett. B 540, 207 (2002).

4. G. Wolf et al., Nucl. Phys. A 552, 549 (1993).

5. Z. Rudy et al., Eur. Phys. J. A 15, 303 (2002).

6. P.A. Piroué and A. J. S. Smith, Phys. Rev. 148, 1315 (1966).

7. K. Tsushima et al., Phys. Rev. C, 59, 369 (1999).

8. G. Fäldt and C. Wilkin, Z. Phys. A 357, 241 (1997).

9. R. Maier, Nucl. Instr. Methods Phys. Res., Sect. A 390, 1 (1997).

10. H. Dombrowski et al., Nucl. Instr. Methods Phys. Res. Sect. A 386, 228 (1997); A. Khoukaz et al., Eur. Phys. J. D 5, 275 (1999).

11. I. Lehmann, Ph.D. thesis, Universität zu Köln (2003); I. Lehmann et al., Nucl. Instr. Methods Phys. Res., Sect. $A$ (in preparation).

12. M. Büscher et al., Nucl. Instr. Methods Phys. Res., Sect. A 481, 378 (2002).

13. V. Koptev et al., JETP 67, 2177 (1988).

14. V.V. Abaev et al., J. Phys. G 14, 903 (1988).

15. J. Papp et al., Phys. Rev. Lett. 34, 601 (1975).

16. K. Hagiwara et al. (Particle Data Group), Phys. Rev. D 66, 010001 (2002).

17. V. Kleber et al., Phys. Rev. Lett. 91, 172304 (2003).

18. D.R.F. Cochran et al., Phys. Rev. D 6, 3085 (1972).

19. M. Büscher et al., Phys. Rev. C 65, 014603 (2001).

20. W. Cassing et al., Phys. Lett. B 238, 25 (1990).

21. H. Müller and K. Sistemich, Z. Phys. A 344, 197 (1992).

22. A.A. Sibirtsev and M. Büscher, Z. Phys. A 347, 191 (1994).

23. E.Ya. Paryev, Eur. Phys. J. A 5, 307 (1999).

24. W. Cassing and E. Bratkovskaya, Phys. Rep. 308, 65 (1999).

25. A. Sibirtsev and W. Cassing, Nucl. Phys. A 641, 476 (1998).

26. Z. Rudy et al., (in preparation).

27. S. Schnetzer et al., Phys. Rev. C 40, 640 (1989).

28. M. Debowski et al., Z. Phys. A 356, 313 (1996).

29. A. Badalà et al., Phys. Rev. Lett. 80, 4863 (1998).

30. A.V. Akindinov et al., JETP Lett. 72, 150 (2000).

31. M. Büscher et al., Z. Phys. A 355, 93 (1996).

32. W. Scheinast, KaoS Collaboration, Proc. $7^{\text {th }}$ Int. Workshop on Meson Production, Properties and Interaction MESON2002, 24 - 28 May 2002, Cracow, Poland; World Scientific Publishing, ISBN 981-238-160-0, L. Jarczyk, A. Magiera, C. Guaraldo, H. Machner, (editors), p. 493 (2003); and private communication. 
33. A.B. Kaidalov and L.A. Kondratyuk, Nucl. Phys. B 57, Appendix 100 (1973); A.B. Kaidalov, Phys. Rep. 50, 157 (1979).

34. V. Koptev et al., Eur. Phys. J. A 17, 235 (2003).

35. V. Franco and R.J. Glauber, Phys. Rev. 142, 1195 (1966).

36. E. Chiavassa et al., Phys. Lett. B, 337, 192 (1994).

37. A. Baldini et al., Total Cross Sections of High Energy Particles, Vol.12 of Landolt-Börnstein, Numerical Data and Functional Relationships in Science and Technology, edited by H. Schopper (Springer-Verlag, Berlin, 1988).

38. http://www-hades.gsi.de/computing/pluto/html/PlutoIndex.htm.

39. M. Büscher, Proc. XXVIII Mazurian Lakes Conference on Physics, Atomic Nucleus as a Laboratory for Fundamental Processes, 31 August - 7 September 2003, Krzyze, Poland; Acta Physica Polonica, in print; arXiv:nucl-ex/0311018. 
Table 4. Double differential cross sections for $p^{12} \mathrm{C} \rightarrow K^{+} X$ reactions obtained with the $1.3 \mathrm{~T}$ mode. The errors do not include the systematic uncertainties from the normalisation to pion data.

\begin{tabular}{ccccc}
\hline$p_{K^{+}}$ & \multicolumn{4}{c}{$\mathrm{d}^{2} \sigma / \mathrm{d} \Omega \mathrm{d} p(\mathrm{nb} /(\mathrm{sr} \mathrm{MeV} / \mathrm{c}))$} \\
$(\mathrm{MeV} / \mathrm{c})$ & $T_{p}=1.0 \mathrm{GeV}$ & $1.2 \mathrm{GeV}$ & $2.0 \mathrm{GeV}$ & $2.3 \mathrm{GeV}$ \\
\hline $171 \pm 11$ & $0.073 \pm 0.027$ & $0.60 \pm 0.18$ & $9.9 \pm 1.8$ & $11.9 \pm 2.9$ \\
$192 \pm 12$ & $0.073 \pm 0.048$ & $0.88 \pm 0.19$ & $18.6 \pm 1.8$ & $27.9 \pm 4.3$ \\
$216 \pm 11$ & $0.125 \pm 0.028$ & $1.41 \pm 0.23$ & $24.7 \pm 2.1$ & $45.6 \pm 5.1$ \\
$240 \pm 12$ & $0.132 \pm 0.032$ & $1.46 \pm 0.21$ & $28.3 \pm 2.3$ & $51.4 \pm 5.4$ \\
$266 \pm 14$ & $0.178 \pm 0.052$ & $2.47 \pm 0.26$ & $45.7 \pm 2.9$ & $75.5 \pm 6.8$ \\
$295 \pm 15$ & $0.270 \pm 0.036$ & $2.73 \pm 0.28$ & $54.1 \pm 3.0$ & $89.9 \pm 6.7$ \\
$325 \pm 15$ & $0.337 \pm 0.045$ & $4.82 \pm 0.41$ & $86.0 \pm 5.4$ & $138 \pm 12$ \\
$355 \pm 15$ & $0.302 \pm 0.036$ & $6.02 \pm 0.54$ & $99.8 \pm 6.8$ & $158 \pm 13$ \\
$385 \pm 15$ & $0.330 \pm 0.053$ & $6.76 \pm 0.52$ & $115.9 \pm 7.5$ & $188 \pm 15$ \\
$415 \pm 15$ & $0.257 \pm 0.038$ & $6.89 \pm 0.61$ & $145 \pm 11$ & $245 \pm 22$ \\
$445 \pm 15$ & $0.201 \pm 0.066$ & $9.61 \pm 0.85$ & $186 \pm 14$ & $310 \pm 28$ \\
$475 \pm 15$ & $0.165 \pm 0.047$ & $8.21 \pm 0.61$ & $194.4 \pm 9.5$ & $313 \pm 23$ \\
$507 \pm 17$ & $0.088 \pm 0.042$ & $6.44 \pm 0.73$ & $220 \pm 12$ & $389 \pm 30$ \\
\hline
\end{tabular}

Table 5. Double differential cross sections for $p^{12} \mathrm{C} \rightarrow K^{+} X$ reactions obtained with the 1.6 T mode. The errors do not include the systematic uncertainties from the normalisation to pion data.

\begin{tabular}{cccc}
\hline$p_{K^{+}}$ & \multicolumn{3}{c}{$\mathrm{d}^{2} \sigma / \mathrm{d} \Omega \mathrm{d} p(\mathrm{nb} /(\mathrm{sr} \mathrm{MeV} / \mathrm{c}))$} \\
$(\mathrm{MeV} / \mathrm{c})$ & $T_{p}=1.2 \mathrm{GeV}$ & $1.5 \mathrm{GeV}$ & $2.3 \mathrm{GeV}$ \\
\hline $209 \pm 6$ & $1.28 \pm 0.40$ & $14.0 \pm 2.9$ & $73.2 \pm 9.7$ \\
$233 \pm 6$ & $2.07 \pm 0.50$ & $14.8 \pm 2.7$ & $78 \pm 11$ \\
$259 \pm 6$ & $2.82 \pm 0.58$ & $17.1 \pm 3.3$ & $105 \pm 17$ \\
$285 \pm 6$ & $3.51 \pm 0.58$ & $21.5 \pm 3.2$ & $128 \pm 16$ \\
$315 \pm 6$ & $5.52 \pm 0.77$ & $42.0 \pm 5.0$ & $164 \pm 17$ \\
$346 \pm 6$ & $6.41 \pm 0.81$ & $48.5 \pm 5.6$ & $238 \pm 24$ \\
$378 \pm 6$ & $6.96 \pm 0.70$ & $58.0 \pm 5.5$ & $264 \pm 22$ \\
$415 \pm 6$ & $8.29 \pm 0.70$ & $68.2 \pm 5.6$ & $321 \pm 23$ \\
$450 \pm 6$ & $7.94 \pm 0.79$ & $77.1 \pm 7.4$ & $351 \pm 31$ \\
$486 \pm 6$ & $7.24 \pm 0.59$ & $84.4 \pm 6.7$ & $373 \pm 27$ \\
$521 \pm 6$ & $6.88 \pm 0.53$ & $87.8 \pm 6.5$ & $416 \pm 26$ \\
$557 \pm 6$ & $6.09 \pm 0.73$ & $99 \pm 11$ & $491 \pm 48$ \\
$595 \pm 6$ & $4.40 \pm 0.55$ & $82.2 \pm 8.4$ & $428 \pm 38$ \\
\hline
\end{tabular}

Table 6. Cross section ratios $A / \mathrm{C}$ measured at $T_{p}=1.0 \mathrm{GeV}$ with the $1.3 \mathrm{~T}$ mode. The error bars include the statistical and systematic uncertainties.

\begin{tabular}{ccc}
\hline$p_{K^{+}}(\mathrm{MeV} / \mathrm{c})$ & \multicolumn{2}{c}{ Ratio } \\
& $\mathrm{Cu} / \mathrm{C}$ & $\mathrm{Au} / \mathrm{C}$ \\
\hline $216 \pm 11$ & $2.05 \pm 1.30$ & $4.65 \pm 3.60$ \\
$240 \pm 12$ & $3.60 \pm 1.25$ & $5.70 \pm 3.30$ \\
$266 \pm 14$ & $3.40 \pm 1.10$ & $5.35 \pm 2.55$ \\
$295 \pm 15$ & $3.95 \pm 0.85$ & $6.20 \pm 1.90$ \\
$325 \pm 15$ & $3.85 \pm 1.00$ & $6.20 \pm 2.05$ \\
$355 \pm 15$ & $4.10 \pm 0.95$ & $7.40 \pm 2.05$ \\
$385 \pm 15$ & $4.65 \pm 0.95$ & $8.25 \pm 2.10$ \\
$415 \pm 15$ & $4.95 \pm 0.95$ & $9.10 \pm 2.30$ \\
$445 \pm 15$ & $5.15 \pm 1.50$ & $7.40 \pm 3.20$ \\
$475 \pm 15$ & $4.10 \pm 1.30$ & $5.50 \pm 2.55$ \\
$507 \pm 17$ & $4.70 \pm 2.10$ & $10.55 \pm 7.15$ \\
\hline
\end{tabular}


Table 7. Cross section ratios $\mathrm{Au} / \mathrm{C}$ measured at $T_{p}=1.5 \mathrm{GeV}$ with the $1.6 \mathrm{~T}$ mode. The error bars include the statistical and systematic uncertainties.

\begin{tabular}{cc}
\hline$p_{K^{+}}(\mathrm{MeV} / \mathrm{c})$ & $\begin{array}{c}\text { Ratio } \\
\mathrm{Au} / \mathrm{C}\end{array}$ \\
\hline $184 \pm 8$ & $3.18 \pm 0.94$ \\
$209 \pm 11$ & $4.75 \pm 0.71$ \\
$233 \pm 11$ & $6.71 \pm 0.61$ \\
$260 \pm 12$ & $6.40 \pm 0.39$ \\
$285 \pm 13$ & $5.68 \pm 0.36$ \\
$315 \pm 14$ & $4.76 \pm 0.27$ \\
$347 \pm 14$ & $4.96 \pm 0.22$ \\
$378 \pm 14$ & $4.42 \pm 0.18$ \\
$412 \pm 14$ & $4.33 \pm 0.17$ \\
$448 \pm 14$ & $4.11 \pm 0.16$ \\
$485 \pm 15$ & $3.82 \pm 0.15$ \\
$522 \pm 15$ & $3.70 \pm 0.18$ \\
$560 \pm 16$ & $3.74 \pm 0.23$ \\
$596 \pm 16$ & $3.88 \pm 0.27$ \\
\hline
\end{tabular}

Table 8. Cross section ratios $\mathrm{Au} / \mathrm{C}$ measured at $T_{p}=1.75 \mathrm{GeV}$ with the $1.6 \mathrm{~T}$ mode. The error bars include the statistical and systematic uncertainties.

\begin{tabular}{cc}
\hline$p_{K^{+}}(\mathrm{MeV} / \mathrm{c})$ & $\begin{array}{c}\text { Ratio } \\
\mathrm{Au} / \mathrm{C}\end{array}$ \\
\hline $158 \pm 8$ & $1.96 \pm 2.00$ \\
$184 \pm 8$ & $4.00 \pm 1.41$ \\
$209 \pm 11$ & $6.24 \pm 0.73$ \\
$233 \pm 11$ & $9.61 \pm 1.02$ \\
$260 \pm 12$ & $8.15 \pm 0.49$ \\
$285 \pm 12$ & $7.01 \pm 0.42$ \\
$315 \pm 13$ & $6.23 \pm 0.33$ \\
$347 \pm 14$ & $5.38 \pm 0.24$ \\
$378 \pm 14$ & $4.83 \pm 0.20$ \\
$412 \pm 14$ & $4.65 \pm 0.18$ \\
$448 \pm 15$ & $4.64 \pm 0.18$ \\
$485 \pm 15$ & $4.19 \pm 0.16$ \\
$522 \pm 15$ & $4.12 \pm 0.18$ \\
$560 \pm 16$ & $3.85 \pm 0.21$ \\
$596 \pm 16$ & $3.76 \pm 0.24$ \\
\hline
\end{tabular}


Table 9. Cross section ratios $A / \mathrm{C}$ measured at $T_{p}=2.3 \mathrm{GeV}$ and different $\mathrm{D} 2$ operation modes. The error bars include the statistical and systematic uncertainties.

\begin{tabular}{|c|c|c|c|c|}
\hline \multicolumn{2}{|c|}{$p_{K^{+}}(\mathrm{MeV} / \mathrm{c})$} & \multicolumn{3}{|c|}{ Ratio } \\
\hline$B=1.3 \mathrm{~T}$ & $1.6 \mathrm{~T}$ & $\mathrm{Cu} / \mathrm{C}$ & $\mathrm{Ag} / \mathrm{C}$ & $\mathrm{Au} / \mathrm{C}$ \\
\hline \multirow[t]{2}{*}{$153 \pm 9$} & & $2.54 \pm 0.30$ & $2.62 \pm 0.33$ & \\
\hline & $158 \pm 8$ & & $3.62 \pm 1.11$ & $2.98 \pm 0.82$ \\
\hline \multirow[t]{2}{*}{$175 \pm 11$} & & $3.63 \pm 0.28$ & $4.21 \pm 0.29$ & $5.40 \pm 2.25$ \\
\hline & $182 \pm 8$ & & $5.53 \pm 0.53$ & $4.96 \pm 0.45$ \\
\hline \multirow[t]{2}{*}{$196 \pm 11$} & & $3.86 \pm 0.17$ & $5.03 \pm 0.22$ & $7.44 \pm 0.89$ \\
\hline & $208 \pm 11$ & & $6.17 \pm 0.31$ & $8.24 \pm 0.38$ \\
\hline \multirow[t]{2}{*}{$218 \pm 11$} & & $4.16 \pm 0.14$ & $6.04 \pm 0.20$ & $9.14 \pm 0.72$ \\
\hline & $233 \pm 11$ & & $5.76 \pm 0.25$ & $9.95 \pm 0.40$ \\
\hline \multirow[t]{2}{*}{$239 \pm 11$} & & $3.86 \pm 0.13$ & $6.07 \pm 0.20$ & $10.84 \pm 0.72$ \\
\hline & $259 \pm 12$ & & $5.77 \pm 0.19$ & $10.05 \pm 0.34$ \\
\hline \multirow[t]{2}{*}{$264 \pm 12$} & & $3.71 \pm 0.12$ & $5.67 \pm 0.18$ & $9.31 \pm 0.60$ \\
\hline & $285 \pm 12$ & & $5.25 \pm 0.18$ & $8.57 \pm 0.29$ \\
\hline \multirow[t]{2}{*}{$293 \pm 14$} & & $3.57 \pm 0.10$ & $5.14 \pm 0.14$ & $7.91 \pm 0.47$ \\
\hline & $315 \pm 13$ & & $4.59 \pm 0.15$ & $7.62 \pm 0.25$ \\
\hline $317 \pm 14$ & & $3.228 \pm 0.084$ & $4.51 \pm 0.12$ & $7.18 \pm 0.43$ \\
\hline \multirow[t]{2}{*}{$345 \pm 15$} & & $3.155 \pm 0.083$ & $4.28 \pm 0.12$ & $6.29 \pm 0.34$ \\
\hline & $350 \pm 14$ & & $4.54 \pm 0.13$ & $6.75 \pm 0.20$ \\
\hline \multirow[t]{2}{*}{$376 \pm 15$} & & $3.032 \pm 0.076$ & $4.14 \pm 0.11$ & $6.16 \pm 0.34$ \\
\hline & $380 \pm 14$ & & $4.20 \pm 0.11$ & $6.31 \pm 0.18$ \\
\hline \multirow[t]{2}{*}{$407 \pm 15$} & & $3.010 \pm 0.080$ & $3.94 \pm 0.11$ & $5.27 \pm 0.30$ \\
\hline & $413 \pm 14$ & & $4.00 \pm 0.10$ & $5.74 \pm 0.16$ \\
\hline \multirow[t]{2}{*}{$438 \pm 15$} & & $2.702 \pm 0.079$ & $3.70 \pm 0.11$ & $4.59 \pm 0.34$ \\
\hline & $450 \pm 14$ & & $3.73 \pm 0.10$ & $5.43 \pm 0.15$ \\
\hline \multirow[t]{2}{*}{$468 \pm 15$} & & $2.73 \pm 0.10$ & $3.60 \pm 0.13$ & $5.06 \pm 0.34$ \\
\hline & $487 \pm 15$ & & $3.54 \pm 0.09$ & $4.97 \pm 0.14$ \\
\hline \multirow[t]{4}{*}{$499 \pm 17$} & & $2.49 \pm 0.11$ & $3.48 \pm 0.15$ & $4.63 \pm 0.34$ \\
\hline & $525 \pm 15$ & & $3.45 \pm 0.10$ & $4.68 \pm 0.14$ \\
\hline & $560 \pm 16$ & & $3.17 \pm 0.10$ & $4.50 \pm 0.14$ \\
\hline & $595 \pm 16$ & & $3.26 \pm 0.11$ & $4.45 \pm 0.15$ \\
\hline
\end{tabular}


Table 10. Double differential cross section for $p \mathrm{D} \rightarrow K^{+} X$ reactions measured at 1.83 and $2.02 \mathrm{GeV}$ with the $1.45 \mathrm{~T}$ and $1.6 \mathrm{~T}$ modes, respectively. The errors do not include the overall $20 \%$ systematic uncertainty from the determination of the luminosity.

\begin{tabular}{|c|c|c|c|}
\hline \multicolumn{2}{|c|}{$p_{K^{+}}(\mathrm{MeV} / \mathrm{c})$} & \multicolumn{2}{|c|}{$\mathrm{d}^{2} \sigma / \mathrm{d} \Omega \mathrm{d} p(\mathrm{nb} /(\mathrm{sr} M e V / \mathrm{c}))$} \\
\hline$B=1.45 \mathrm{~T}$ & $1.6 \mathrm{~T}$ & $T_{p}=1.83 \mathrm{GeV}$ & $2.02 \mathrm{GeV}$ \\
\hline $212 \pm 12$ & & $2.01 \pm 0.56$ & \\
\hline & $212 \pm 12$ & & $5.4 \pm 1.2$ \\
\hline $236 \pm 16$ & & $2.34 \pm 0.56$ & \\
\hline & $237 \pm 12$ & & $7.2 \pm 1.4$ \\
\hline $261 \pm 16$ & & $5.0 \pm 1.1$ & \\
\hline & $262 \pm 12$ & & $11.6 \pm 2.5$ \\
\hline $287 \pm 12$ & & $5.21 \pm 0.96$ & \\
\hline $319 \pm 15$ & $289 \pm 13$ & $6.49+0.87$ & $16.2 \pm 2.7$ \\
\hline & $319 \pm 15$ & & $16.4 \pm 2.9$ \\
\hline $349 \pm 17$ & & $10.5 \pm 1.2$ & \\
\hline & $350 \pm 16$ & & $28.8 \pm 2.8$ \\
\hline $382 \pm 14$ & & $14.5 \pm 1.7$ & \\
\hline & $381 \pm 15$ & & $32.9 \pm 3.9$ \\
\hline $415 \pm 15$ & $413 \pm 15$ & $15.8 \pm 1.7$ & $42.9 \pm 4.2$ \\
\hline $449 \pm 17$ & & $19.1 \pm 1.7$ & \\
\hline & $449 \pm 17$ & & $47.5 \pm 4.1$ \\
\hline $485 \pm 17$ & & $22.2 \pm 1.9$ & \\
\hline & $484 \pm 17$ & & $52.9 \pm 5.0$ \\
\hline $521 \pm 18$ & & $33.0 \pm 3.9$ & \\
\hline & $519 \pm 17$ & & $60.8 \pm 7.0$ \\
\hline $554 \pm 12$ & & $29.0 \pm 3.4$ & \\
\hline & $555 \pm 18$ & & $70.0 \pm 7.5$ \\
\hline $587 \pm 15$ & $591 \pm 18$ & $29.1 \pm 3.4$ & $68.6 \pm 7.8$ \\
\hline
\end{tabular}

Table 11. Relevant reaction channels for $K^{+}$production on deuterons and the corresponding total cross sections calculated according to the parameterisation taken from Ref. [7].

\begin{tabular}{ccc}
\hline & \multicolumn{2}{c}{$\sigma(\mu \mathrm{b})$} \\
Reaction & $T_{p}=1.83 \mathrm{GeV}$ & $2.02 \mathrm{GeV}$ \\
\hline$p p \rightarrow \Lambda K^{+} p$ & 3.85 & 10.9 \\
$p n \rightarrow \Lambda K^{+} n$ & 7.12 & 19.4 \\
$p p \rightarrow \Sigma^{0} K^{+} p$ & 0.005 & 1.36 \\
$p n \rightarrow \Sigma^{0} K^{+} n$ & 0.008 & 1.68 \\
$p p \rightarrow \Sigma^{+} K^{+} n$ & 0.001 & 0.52 \\
$n p \rightarrow \Sigma^{-} K^{+} p$ & 0.010 & 2.78 \\
\hline
\end{tabular}

